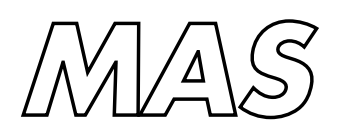

Modelling, Analysis and Simulation equation

C.J. van Duijn, L.A. Peletier, I.S. Pop

Report MAS-E0503 January 2005 
$\mathrm{CWI}$ is the National Research Institute for Mathematics and Computer Science. It is sponsored by the Netherlands Organization for Scientific Research (NWO).

$\mathrm{CWI}$ is a founding member of ERCIM, the European Research Consortium for Informatics and Mathematics.

CWI's research has a theme-oriented structure and is grouped into four clusters. Listed below are the names of the clusters and in parentheses their acronyms.

Probability, Networks and Algorithms (PNA)

Software Engineering (SEN)

\section{Modelling, Analysis and Simulation (MAS)}

Information Systems (INS)

Copyright (C) 2005, Stichting Centrum voor Wiskunde en Informatica

P.O. Box 94079, 1090 GB Amsterdam (NL)

Kruislaan 413, 1098 SJ Amsterdam (NL)

Telephone +31205929333

Telefax +31205924199

ISSN 1386-3703 


\title{
A new class of entropy solutions of the Buckley- Leverett equation
}

\begin{abstract}
We discuss an extension of the Buckley-Leverett $(B L)$ equation describing two-phase flow in porous media. This extension includes a third order mixed derivatives term and models the dynamic effects in the pressure difference between the two phases. We derive existence conditions for travelling wave solutions of the extended model. This leads to admissible shocks for the original $\mathrm{BL}$ equation, which violate the Oleinik entropy condition and are therefore called nonclassical. In this way we obtain non-monotone weak solutions of the BL problem consisting of steady states separated by shocks, confirming results obtained experimentally.
\end{abstract}

2000 Mathematics Subject Classification: 35K70 Ultraparabolic, pseudoparabolic PDE, 76L05 Shock waves and blast waves, 76S05 Flows in porous media; filtration; seepage, 35L67 Shocks and singularities, 35L65 Conservation laws, 35M10 PDE of mixed type

Keywords and Phrases: conservation laws; dynamic capillary pressure; pseudo-parabolic

Note: This work was carried out under the project PDE@CWI (MAS 1.2) 



\title{
A new class of entropy solutions of the Buckley-Leverett equation
}

\author{
C.J. van Duijn*, L.A. Peletier ${ }^{\dagger} \&$ I.S. Pop*
}

January 12,2005

\section{Introduction}

We consider the first order initial-boundary value problem

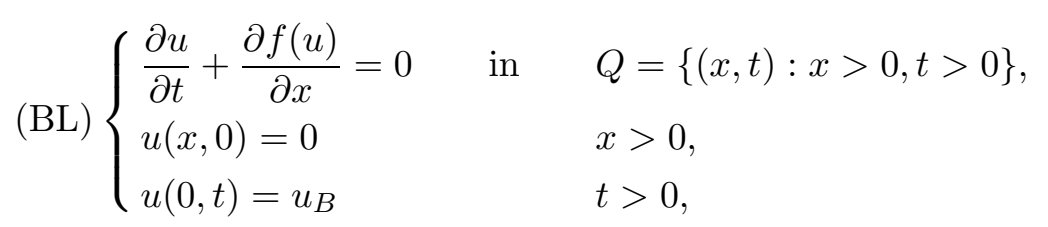

where $u_{B}$ is a constant such that $0 \leq u_{B} \leq 1$. The nonlinearity $f: \mathbf{R} \rightarrow \mathbf{R}$ is given by

$$
f(u)=\frac{u^{2}}{u^{2}+M(1-u)^{2}}, \quad \text { if } \quad 0 \leq u \leq 1
$$

and by $f(u)=0$ if $u<0$ and $f(u)=1$ if $u>1$. It is shown in Figure 1.

Equation (1.1), with the given function $f$, arises in two-phase flow in porous media and Problem (BL) models oil recovery by water-drive in one-dimensional horizontal flow. In this context, $u: \bar{Q} \rightarrow[0,1]$ denotes water saturation, $f$ the water fractional flow function and $M$ the water/oil viscosity ratio. In petroleum engineering, equation (1.1) is known as the Buckley-Leverett equation [3]. It is a prototype for first-order conservation laws with convex-concave flux functions.

It is well known that first-order equations such as (1.1) may have solutions with discontinuities, or shocks. The value $\left(u_{\ell}\right)$ to the left of the shock, the value $\left(u_{r}\right)$ to the right, and the speed $s$ of the shock with trace $x=x(t)$ are related through the Rankine-Hugoniot condition,

$$
\frac{d x}{d t}=s=\frac{f\left(u_{\ell}\right)-f\left(u_{r}\right)}{u_{\ell}-u_{r}}
$$

* Department of Mathematics and Computer Science, Technische Universiteit Eindhoven, PO Box 513, 5600 MB Eindhoven, The Netherlands \{c.j.v.duijn, i.pop\}@tue.nl.

${ }^{\dagger}$ Mathematical Institute, Leiden University, PO Box 9512, 2300 RA Leiden, The Netherlands peletier@math.leidenuniv.nl, and Centrum voor Wiskunde en Informatica, PB 94079, 1090 GB Amsterdam, The Netherlands. 


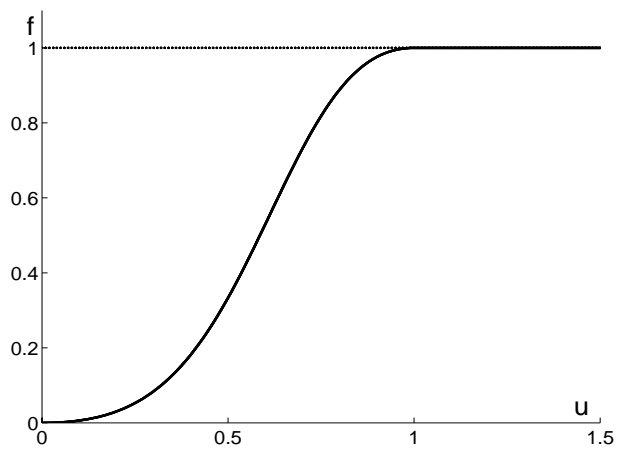

Figure 1: Nonlinear flux function for Buckley-Leverett $(M=2)$

We will denote shocks by their values to the left and to the right: $\left\{u_{\ell}, u_{r}\right\}$.

If a function $u$ is such that equation (1.1) is satisfied away from the shock curve, and the Rankine-Hugoniot condition is satisfied across the curve, then $u$ satisfies the identity

$$
\int_{Q}\left\{u \frac{\partial \varphi}{\partial t}+f(u) \frac{\partial \varphi}{\partial x}\right\}=0 \quad \text { for all } \quad \varphi \in C_{0}^{\infty}(Q)
$$

Functions $u \in L^{\infty}(Q)$, which satisfy (1.4) are called weak solutions of equation (1.1). Clearly, for any $u_{B} \in[0,1]$, a weak solution of Problem (BL) is given by the shock wave

$$
u(x, t)=S(x, t) \stackrel{\text { def }}{=}\left\{\begin{array}{lll}
u_{B} & \text { for } & x<s t \\
0 & \text { for } & x>s t
\end{array} \quad \text { where } \quad s=\frac{f\left(u_{B}\right)}{u_{B}} .\right.
$$

Equation (1.1) usually arises as the limit of a family of extended equations of the form

$$
\frac{\partial u}{\partial t}+\frac{\partial f(u)}{\partial x}=\mathcal{A}_{\varepsilon}(u), \quad \varepsilon>0
$$

in which $\mathcal{A}_{\varepsilon}(u)$ is a singular regularisation term involving higher order derivatives. It is often referred to as a viscosity term. Weak solutions of Problem (BL) are called admissible when they can be constructed as limits, as $\varepsilon \rightarrow 0$, of solutions $u_{\varepsilon}$ of equation (1.6), i.e., for which $\mathcal{A}_{\varepsilon}\left(u_{\varepsilon}\right) \rightarrow 0$ as $\varepsilon \rightarrow 0$ in some weak sense. We return to this limit in Section 6 . This raises the question which of the shock waves $S(x, t)$ defined in (1.5) are admissible. We shall see that this depends on the operator $\mathcal{A}_{\varepsilon}$. To obtain criteria for admissibility we shall use families of travelling wave solutions.

A classical viscosity term is

$$
\mathcal{A}_{\varepsilon}(u)=\varepsilon \frac{\partial^{2} u}{\partial x^{2}}
$$

and with this term, equation (1.6) becomes

$$
\frac{\partial u}{\partial t}+\frac{\partial f(u)}{\partial x}=\varepsilon \frac{\partial^{2} u}{\partial x^{2}} .
$$

Seeking a travelling wave solution, we put

$$
u=u(\eta) \quad \text { with } \quad \eta=\frac{x-s t}{\varepsilon},
$$


and we find that $u(\eta)$ satisfies the following two-point boundary value problem:

$$
\left\{\begin{array}{l}
-s u^{\prime}+(f(u))^{\prime}=u^{\prime \prime} \quad \text { in } \quad \mathbf{R} \\
u(-\infty)=u_{\ell}, \quad u(\infty)=u_{r},
\end{array}\right.
$$

where primes denote differentiation with respect to $\eta$. An elementary analysis shows that Problem (1.9) has a solution if and only if $f$ and the limiting values $u_{\ell}$ and $u_{r}$ satisfy $(i)$ the Rankine-Hugoniot condition (1.3), and (ii) the Oleinik entropy condition [19]:

$$
\frac{f\left(u_{\ell}\right)-f(u)}{u_{\ell}-u} \geq \frac{f\left(u_{\ell}\right)-f\left(u_{r}\right)}{u_{\ell}-u_{r}} \quad \text { for } u \text { between } u_{\ell} \text { and } u_{r}
$$

Note that in the limit as $\varepsilon \rightarrow 0^{+}$, travelling waves converge to the shock $\left\{u_{\ell}, u_{r}\right\}$.

Applying $(\mathrm{RH})$ and $(\mathrm{E})$ to the flux function (1.2) we find that the function $S(x, t)$ defined in (1.5) is an admissible shock wave if and only if

$$
s=\frac{f\left(u_{B}\right)}{u_{B}} \quad(\mathrm{RH}) \quad \text { and } \quad u_{B} \leq \alpha \quad(\mathrm{E}),
$$

where $\alpha$ is the unique root of the equation

$$
f^{\prime}(u)=\frac{f(u)}{u} .
$$

It is found to be given by

$$
\alpha=\sqrt{\frac{M}{M+1}} .
$$

Clearly, $0<\alpha<1$.

Remark. Shocks $\left\{u_{\ell}, u_{r}\right\}$ which satisfy (E) are called classical shocks.

The characteristic speeds to the left and to the right of the shock are given by, respectively, $f^{\prime}\left(u_{l}\right)$ and $f^{\prime}\left(u_{r}\right)$. It can be seen by inspection of the graph of $f$ that

$$
f^{\prime}\left(u_{B}\right)>s>f^{\prime}(0) \quad \text { if } \quad 0<u_{B}<\alpha
$$

and

$$
s>f^{\prime}\left(u_{B}\right) \geq f^{\prime}(0) \quad \text { if } \quad \alpha<u_{B}<\infty .
$$

Thus, if $u_{B} \in(0, \alpha)$, then characteristics run into the shock from both sides, whilst if $u_{B}>\alpha$, characteristics run into the shock at the front, but not at the back. We see that in this example the admissibility condition (E) coincides with the Lax admissibility condition for convex fluxes, which states that a shock is admissible if

$$
f^{\prime}\left(u_{l}\right)>s>f^{\prime}\left(u_{r}\right) .
$$

One then speaks of a Lax discontinuity ([17], p. 119). 
In recent years other choices for the viscosity term $\mathcal{A}_{\varepsilon}$ have been investigated. We mention the extension introduced in [17] (cf p. 18 and 53ff) which contains, besides a diffusive term as in (1.7), also a dispersive term, and results in the equation

$$
\frac{\partial u}{\partial t}+\frac{\partial u^{3}}{\partial x}=\varepsilon \frac{\partial^{2} u}{\partial x^{2}}+\varepsilon^{2} a \frac{\partial^{3} u}{\partial x^{3}}, \quad a \in \mathbf{R}^{+} .
$$

and the work of Bertozzi, Münch and Shearer [4], [5] which involves a fourth order extension motivated by the Thin Film Equation:

$$
\frac{\partial u}{\partial t}+\frac{\partial}{\partial x}\left(u^{2}-u^{3}\right)=-\varepsilon^{3} \frac{\partial}{\partial x}\left(u^{3} \frac{\partial^{3} u}{\partial x^{3}}\right) .
$$

It is found that for certain combinations of the parameters involved, shock waves are admissible for which the classical entropy condition (E) is violated. Specifically, in some instances, shock waves may be undercompressive [17], which means that both conditions (E) and (L) are violated in the sense that

$$
s>f^{\prime}\left(u_{l}\right)>f^{\prime}\left(u_{r}\right)
$$

In this paper we discuss an extension which is motivated by the theory of two-phase flow in porous media. In this context, the viscous term $\mathcal{A}_{\varepsilon}$ models capillary effects between the phases and builds upon an expression for the difference between the oil and water pressure. In the classical approach, e.g. [3], this pressure difference is considered to be a unique function of the water saturation, the so-called capillary pressure. Simplifying to linear terms this yields the parabolic extension (1.7).

However, in the past decades it has been recognized that the pressure difference between the phases is not a unique function of the saturation [18], but involves hysteretic and dynamic effects [20]. Theoretical studies [14], [15] based on thermodynamic considerations, show the occurrence of the time derivative of the saturation as well as the capillary pressure relation in the phase pressure difference. Restricting to linear terms, this leads to the pseudo-parabolic equation

$$
\frac{\partial u}{\partial t}+\frac{\partial f(u)}{\partial x}=\varepsilon \frac{\partial^{2} u}{\partial x^{2}}+\varepsilon^{2} \tau \frac{\partial^{3} u}{\partial x^{2} \partial t}, \quad \tau \in \mathbf{R}^{+} .
$$

We derive existence conditions for traveling wave solutions of (1.17) and so obtain admissibility conditions for shocks $\left\{u_{\ell}, u_{r}\right\}$ of (1.1) which will be used to solve Problem (BL). Specifically, we find fast undercompressive shocks for which (1.16) holds, and thus violates condition (E), and we find weak solutions of Problem (BL), which consist of constant states separated by shocks, which are not monotone. This confirms what is found in experiments [10].

We derive admissibility conditions of shocks by analyzing the existence and the nonexistence of traveling wave solutions of equation (1.17) with appropriate limit values $u_{\ell}$ and $u_{r}$. For a given value of the constant $M$ in $f$, the parameter $\tau$ will be seen to serve as a bifurcation parameter: for small values of $\tau>0$ the situation will be much like in the classical case (E), but when $\tau$ exceeds a critical value $\tau_{*}>0$ the situation changes 
abruptly and new types of shock waves become admissible. In the following three theorems we give conditions for the existence and nonexistence of traveling wave solutions of equation (1.17) which form the basis of classical and nonclassical admissibility conditions for equation (1.1). In the next section, they will be used to construct admissible weak solutions of Problem (BL).

Substituting (1.8) into (1.17) we obtain the equation

$$
-s u^{\prime}+(f(u))^{\prime}=u^{\prime \prime}-s \tau u^{\prime \prime \prime} \quad \text { in } \quad \mathbf{R} .
$$

When we integrate this equation over $(\eta, \infty)$, we obtain the second order boundary value problem

$$
(\mathrm{TW})\left\{\begin{array}{l}
-s\left(u-u_{r}\right)+\left\{f(u)-f\left(u_{r}\right)\right\}=u^{\prime}-s \tau u^{\prime \prime} \quad \text { in } \quad \mathbf{R}, \\
u(-\infty)=u_{\ell}, \quad u(\infty)=u_{r},
\end{array}\right.
$$

where $s=s\left(u_{\ell}, u_{r}\right)$ is given by the Rankine-Hugoniot condition (1.3).

We consider two cases:

$$
\text { (I) } u_{r}=0, \quad u_{\ell}>0 \text { and (II) } u_{r}>u_{\ell}>0 \text {. }
$$

Case I: $\mathbf{u}_{\mathbf{r}}=\mathbf{0}$ We first establish an upper bound for $u_{\ell}$.

Proposition 1.1 Let $u$ be a solution of Problem $(T W)$ such that $u_{r}=0$. Then, $u_{\ell}<\beta$, where $\beta$ is the value of $u$ for which the equal area rule holds:

$$
\int_{0}^{\beta}\left\{f(u)-\frac{f(\beta)}{\beta} u\right\} d u=0 .
$$

In Figure 2 we indicate the different critical values of $u$ in a graph of $f(u)$ when $M=2$.

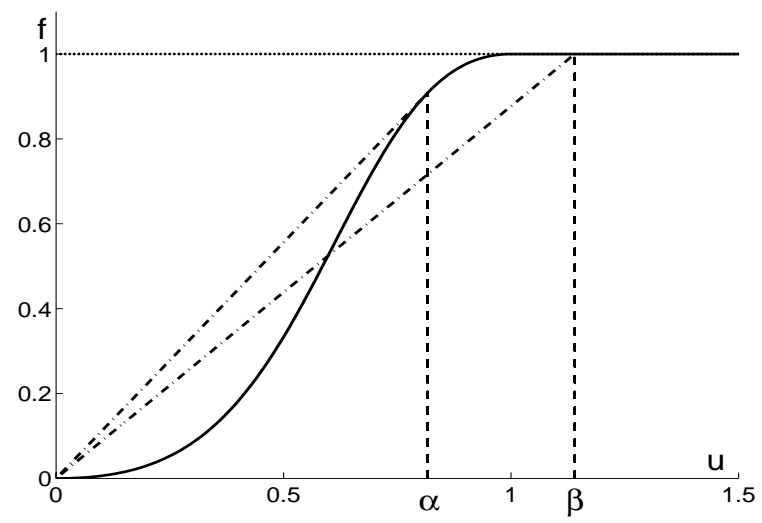

Figure 2: Critical values of $u$ when $M=2: \alpha=0.8164966 \ldots$ and $\beta=1.147745087 \ldots$

Proof. When we put $u_{r}=0$ into (1.18a), multiply by $u^{\prime}$ and integrate over $\mathbf{R}$, we obtain the inequality

$$
\int_{0}^{u_{\ell}}\left\{f(u)-\frac{f\left(u_{\ell}\right)}{u_{\ell}} u\right\} d u=-\int_{\mathbf{R}}\left(u^{\prime}\right)^{2}(\eta) d \eta<0
$$


from which it readily follows that $u_{\ell}<\beta$.

Next, we turn to the questions of existence and uniqueness. Note that if $u_{\ell} \in(\alpha, \beta)$, then

$$
s=s\left(u_{\ell}, 0\right)=\frac{f\left(u_{\ell}\right)}{u_{\ell}}>f^{\prime}\left(u_{\ell}\right) \geq f^{\prime}(0) \quad \text { for } \quad u_{\ell}>\alpha,
$$

and traveling waves - if they exist - lead to an admissibility condition for fast undercompressive waves. For convenience we write $s\left(u_{\ell}, 0\right)=s\left(u_{\ell}\right)$.

In the theorems below we fix $M>0$. We first show that for each $\tau>0$, there exists a unique value of $u_{\ell} \geq \alpha$ - denoted by $\bar{u}(\tau)$ - for which there exists a solution of Problem (TW) such that $u_{r}=0$.

Theorem 1.1 Let $M>0$ be given. Then there exists a constant $\tau_{*}>0$ such that:

(a) for every $0 \leq \tau \leq \tau_{*}$, Problem (TW) has a unique solution with $u_{\ell}=\alpha$ and $u_{r}=0$.

(b) for each $\tau>\tau_{*}$ there exists a unique constant $\bar{u}_{\ell}(\tau) \in(\alpha, \beta)$ such that Problem $(T W)$ has a unique solution with $u_{\ell}=\bar{u}_{\ell}(\tau)$ and $u_{r}=0$.

(c) the function $\bar{u}:[0, \infty) \rightarrow[\alpha, \beta)$ defined by

$$
\bar{u}(\tau)=\left\{\begin{array}{lll}
\alpha & \text { for } & 0 \leq \tau \leq \tau_{*} \\
\bar{u}_{\ell}(\tau) & \text { for } \quad \tau>\tau_{*}
\end{array}\right.
$$

is continuous, strictly increasing for $\tau \geq \tau_{*}$, and $\bar{u}(\infty)=\beta$.

The solutions in Parts (a) and (b) are strictly decreasing.

We shall refer to $\bar{u}=\bar{u}(\tau)$ as the plateau value of $u$. In the sequel we shall often denote the speed $s(\bar{u})$ of the shock $\{\bar{u}, 0\}$ by $\bar{s}$.

Next, suppose that $u_{\ell} \neq \bar{u}(\tau)$. To deal with this case we need to introduce another critical value of $u$, which we denote by $\underline{u}(\tau)$.

- For $\tau \in\left[0, \tau_{*}\right]$ we put $\underline{u}(\tau)=\alpha$, and

- For $\tau>\tau_{*}$ we define $\underline{u}(\tau)$ as the unique zero in the interval $(0, \bar{u}(\tau))$ of the equation

$$
f(r)-\frac{f(\bar{u})}{\bar{u}} r=0, \quad 0<r<\bar{u} .
$$

Plainly, if $\tau>\tau_{*}$, then

$$
0<\underline{u}(\tau)<\alpha<\bar{u}(\tau)<\beta \quad \text { for } \quad \tau>\tau_{*} .
$$

In Figure 3, we show graphs of the functions $\bar{u}(\tau)$ and $\underline{u}(\tau)$. Both graphs have been computed numerically for $M=2$ by applying a shooting technique to a first order problem derived from (4.3), in which $u$ is the independent variable.

The following theorem states that if $u_{r}=0$ and $u_{\ell} \in(0, \bar{u})$, then travelling waves exist if and only if $u_{\ell}<\underline{u}(\tau)$. 


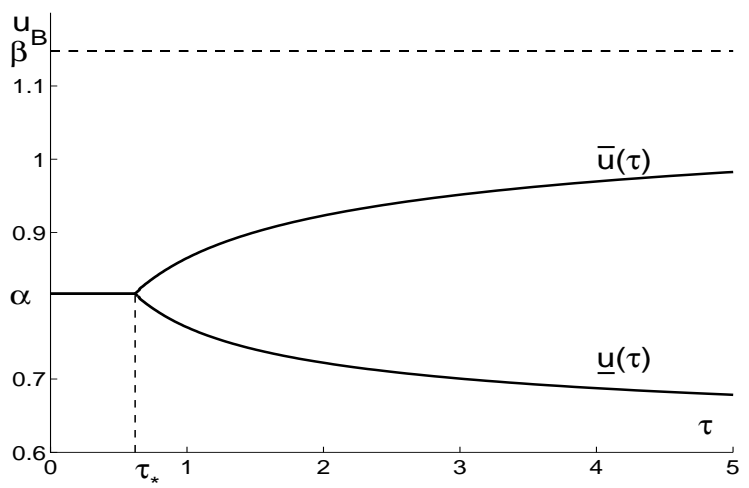

Figure 3: The functions $\bar{u}(\tau)$ and $\underline{u}(\tau)$ computed for $M=2$

Theorem 1.2 Let $M>0$ and $\tau>0$ be given, and let $\underline{u}=\underline{u}(\tau)$ and $\bar{u}=\bar{u}(\tau)$.

(a) For any $u_{\ell} \in(0, \underline{u})$, there exists a unique solution of Problem (TW) such that $u_{r}=0$. We have $s\left(u_{\ell}\right)<\bar{s}$.

(b) Let $\tau>\tau_{*}$. Then for any $u_{\ell} \in(\underline{u}, \bar{u})$, there exists no solution of Problem (TW) such that $u_{r}=0$.

The solution in Part (a) may exhibit a damped oscillation as it tends to $u_{\ell}$.

Case II: $\mathbf{u}_{\mathbf{r}}>\mathbf{0}$ The results of Case I raise the question as to how to deal with solutions of Problem $(\mathrm{BL})$ when $u_{B} \in(\underline{u}, \bar{u})$ and by Theorem 1.2 there is no travelling wave solution with $u_{r}=0$. In this situation we use two travelling waves in succession: one from $u_{B}$ to the plateau value $\bar{u}$, and one from $\bar{u}$ down to $u=0$. The existence of the latter has been established in Theorem 1.1. In the next theorem we deal with the former, in which $u_{r}=\bar{u}$.

Theorem 1.3 Let $M>0$ and $\tau>\tau_{*}$ be given, and let $\underline{u}=\underline{u}(\tau)$ and $\bar{u}=\bar{u}(\tau)$.

(a) For any $u_{\ell} \in(\underline{u}, \bar{u})$, there exists a unique solution of Problem $(T W)$ such that $u_{r}=\bar{u}$. We have $s\left(u_{\ell}, \bar{u}\right)<\bar{s}$.

(b) For any $u_{\ell} \in(0, \underline{u})$, there exists no solution of Problem (TW) such that $u_{r}=\bar{u}$.

The solution in Part (a) may exhibit a damped oscillation as it tends to $u_{\ell}$.

In Section 2 we show how these theorems can be used to construct weak solutions of Problem (BL), i.e. weak solutions, which are admissible within the context of the regularization proposed in equation (1.17), and which involve shocks which may be either classical or nonclassical. In Section 3 we solve the Cauchy Problem for equation (1.17) numerically, starting from a smoothed step function, i.e., $u(x, 0)=u_{B} \tilde{H}(-x)$, where $\tilde{H}(x)$ is a regularized Heaviside function, and $M=2$. We find that for different values of the parameters $u_{B}, \tau$ and $\varepsilon$ the solution converges to solutions constructed in Section 2 as $t \rightarrow \infty$. In Sections 4 and 5 we prove Theorems 1.1, 1.2 and 1.3. The proofs rely on phase plane arguments. It is interesting to note that a similar phase plane structure was recently discussed in [21] in the context of a second order model for highway traffic flow, which may also lead to undercompressive waves. We also mention here a numerical study of traveling 
waves of the original, fully nonlinear, equations of the Hassanizadeh-Gray model [11, 12]. Finally, in Section 6 we discuss the dissipation of the entropy function $u^{2} / 2$ when $u$ is the solution of the Cauchy Problem for equation (1.17).

\section{Entropy solutions of Problem (BL)}

In this section we give a classification of admissible solutions of Problem (BL) based on the "extended viscosity model" (1.17), using the results about traveling wave solutions formulated in Theorems 1.1, 1.2 and 1.3. Before doing that we make a few preliminary observations, and we recall the construction based on the classical model (1.7).

Because equation (1.1) is a first order partial differential equation, and $u_{B}$ is a constant, any solution of Problem (BL) only depends on the combination $x / t$, with shocks, constant states and rarefaction waves as building blocks [19]. The latter are continuous solutions of the form

$$
u(x, t)=r(\zeta) \quad \text { with } \quad \zeta=\frac{x}{t} .
$$

After substitution into (1.1) this yields the equation

$$
\frac{d r}{d \zeta}\left(-\zeta+\frac{d f}{d u}(r(\zeta))\right)=0
$$

Hence, the function $r(\zeta)$ satisfies:

$$
\text { either } \quad r=\text { constant } \quad \text { or } \quad \frac{d f}{d u}(r(\zeta))=\zeta .
$$

When solving Problem (BL), we will combine solutions of equation (2.2) with admissible shocks, i.e. shocks $\left\{u_{\ell}, u_{r}\right\}$ in which $u_{\ell}$ and $u_{r}$ are such that equation (1.6), with the a priori selected and physically relevant viscous extension $\mathcal{A}_{\varepsilon}$ has a traveling wave solution $u(\eta)$ such that $u(\eta) \rightarrow u_{\ell}$ as $\eta \rightarrow-\infty$ and $u(\eta) \rightarrow u_{r}$ as $\eta \rightarrow+\infty$.

In the discussion below we shall choose the constant $M$ in the definition (1.2) of $f$ to be positive and fixed. Though in the physical context in which the viscous extension employed in equation (1.17) was derived, $0 \leq u_{B} \leq 1$, here we shall require that $0 \leq u_{B} \leq \beta$. All solution graphs shown in this section are numerically obtained solutions of equation (1.17). They are expressed in terms of the independent variable $\zeta$ and $t$, i.e.

$$
u(x, t)=w(\zeta, t)
$$

and considered for fixed $\varepsilon>0(=1)$ and for large times $t$. We return to the computational aspects in Section 3.

Before discussing the implications of the viscous extension in (1.17), we recall the construction of classical entropy solutions of Problem (BL). It uses (RH), and the entropy condition (E) which was derived for the diffusive viscous extension used in (1.8). We distinguish two cases:
(a) $\quad 0 \leq u_{B} \leq \alpha \quad$ and
(b) $\quad \alpha<u_{B} \leq \beta$. 
Case (a): $0 \leq u_{B} \leq \alpha$. This case was discussed in the Introduction, where we found that the entropy solution is given by the shock $\left\{u_{B}, 0\right\}$.

Case (b): $\alpha<u_{B} \leq \beta$. In the Introduction we saw that in this case, the shock $\left\{u_{B}, 0\right\}$ is no longer a classical entropy solution. Instead, in this case the entropy solution is a composition of three functions:

$$
u(x, t)=v(\zeta)=\left\{\begin{array}{lll}
u_{B} & \text { for } & 0 \leq \zeta \leq \zeta_{B} \\
r(\zeta) & \text { for } & \zeta_{B} \leq \zeta \leq \zeta_{*} \\
0 & \text { for } & \zeta_{*} \leq \zeta<\infty
\end{array}\right.
$$

where $\zeta_{B}$ and $\zeta_{*}$ are determined by

$$
\zeta_{B}=\frac{d f}{d u}\left(u_{B}\right) \quad \text { and } \quad \zeta_{*}=\frac{d f}{d u}(\alpha)=\frac{f(\alpha)}{\alpha}=s(\alpha),
$$

and $r:\left[\zeta_{B}, \zeta_{*}\right] \rightarrow\left[\alpha, u_{B}\right]$ by the relation

$$
\frac{d f}{d u}(r(\zeta))=\zeta \quad \text { for } \quad \zeta_{B} \leq \zeta \leq \zeta_{*} .
$$

Since $f^{\prime \prime}(u)<0$ for $u \in\left[\alpha, u_{B}\right]$, equation (2.4) has a unique solution, and hence $r(\zeta)$ is well defined. Note that if $u_{B} \geq 1$, then $\zeta_{B}=0$, because $f^{\prime}(u)=0$ if $u \geq 1$.

Solutions corresponding to Case (b) are shown in Figure 4.
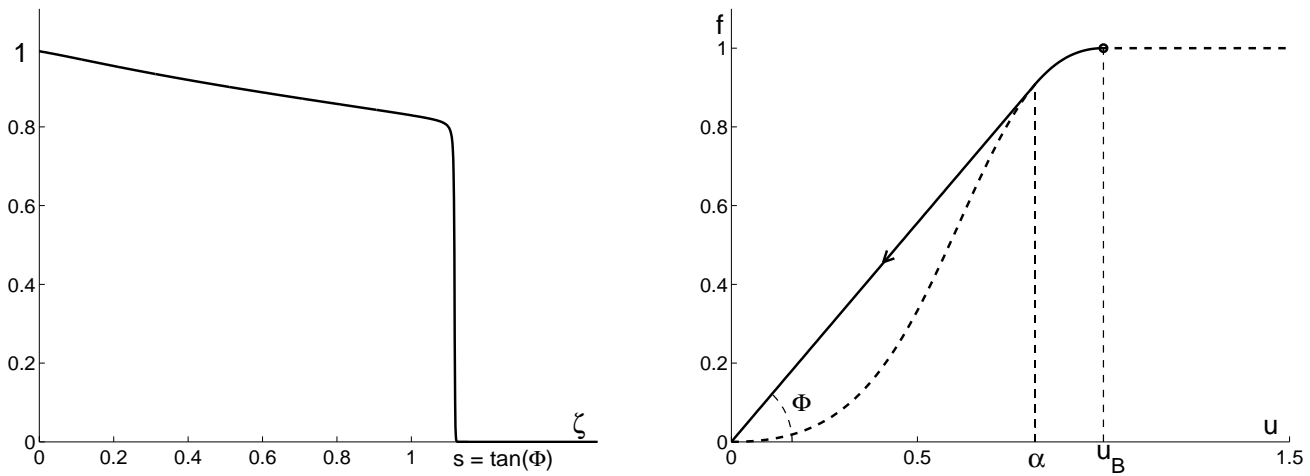

Figure 4: Case (b): Solution graph (left) and flux function with transitions from $u_{B}$ to $\alpha$ and from $\alpha$ to 0 (right)

We now turn to the pseudo-parabolic equation (1.17) that arises in the context of the two-phase flow model of Gray and Hassanizadeh [14], [15]. For this problem, we define a class of non-classical entropy solutions in which shocks are admissible if Problem (TW) has a travelling wave solution with the required limit conditions.

For given $M>0$ and $\tau>0$, the relative values of $u_{B}$ and $\bar{u}(\tau)$ and $\underline{u}(\tau)$ are now important for the type of solution we are going to get. It is easiest to represent them in the $\left(u_{B}, \tau\right)$-plane. Specifically, we distinguish three regions in this plane:

$$
\begin{aligned}
& \mathcal{A}=\left\{\left(u_{B}, \tau\right): \tau>0, \quad \bar{u}(\tau) \leq u_{B}<\beta\right\}, \\
& \mathcal{B}=\left\{\left(u_{B}, \tau\right): \tau>\tau_{*}, \quad \underline{u}(\tau)<u_{B}<\bar{u}(\tau)\right\}, \\
& \mathcal{C}=\left\{\left(u_{B}, \tau\right): \tau>0, \quad 0<u_{B}<\underline{u}(\tau)\right\},
\end{aligned}
$$


These three regions are shown in Figure 5.

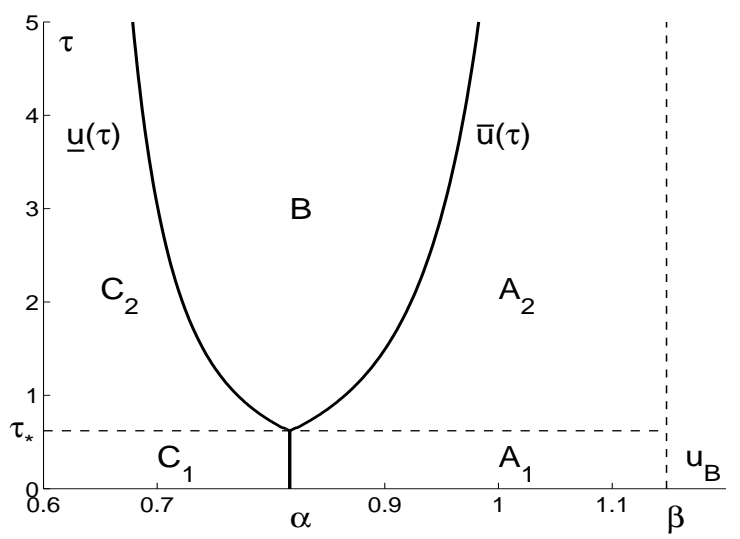

Figure 5: The regions $\mathcal{A}, \mathcal{B}$ and $\mathcal{C}$ in the $\left(u_{B}, \tau\right)$-plane

Case I: $\left(u_{B}, \tau\right) \in \mathcal{A}$

If $0 \leq \tau \leq \tau_{*}$, i.e., $\left(u_{B}, \tau\right) \in \mathcal{A}_{1}$, the construction is as in the classical case described above. After a plateau, where $u=u_{B}$, and $0 \leq \zeta=x / t \leq \zeta_{B}$, we find a rarefaction wave $r(\zeta)$ from $u_{B}$ down to $\alpha$ followed by a classical shock connecting $\alpha$ to the initial state $u=0$.

If $\tau>\tau_{*}$, i.e., $\left(u_{B}, \tau\right) \in \mathcal{A}_{2}$, the solution starts out as before, with a plateau where $u=u_{B}$ and $0<\zeta<\zeta_{B}$, and a rarefaction wave $r(\zeta)$ which now takes $u$ down from $u_{B}$ to $\bar{u}>\alpha$. This takes place over the interval $\zeta_{B} \leq \zeta \leq \bar{\zeta}$. By $(2.2)$,

$$
\bar{\zeta}=\frac{d f}{d u}(\bar{u}(\tau))
$$

Subsequently, $u$ drops down to the initial state $u=0$ through a shock, $\{\bar{u}, 0\}$, which is admissible by Theorem 1.1. By $(\mathrm{RH})$ the shock moves with speed

$$
s=\bar{s}=\frac{f(\bar{u})}{\bar{u}}>\frac{d f}{d u}(\bar{u})=\bar{\zeta}
$$

because $f$ is concave on $(\alpha, \infty)$. Therefore, the shock outruns the rarefaction wave and a second plateau develops between the rarefaction wave and the shock in which $u=\bar{u}$. Summarising, we find that the (non-classical) entropy solution has the form:

$$
u(x, t)=v(\zeta)=\left\{\begin{array}{lll}
u_{B} & \text { for } & 0 \leq \zeta \leq \zeta_{B} \\
r(\zeta) & \text { for } & \zeta_{B} \leq \zeta \leq \bar{\zeta} \\
\bar{u}(\tau) & \text { for } & \bar{\zeta} \leq \zeta \leq \bar{s} \\
0 & \text { for } & \bar{s} \leq \zeta<\infty .
\end{array}\right.
$$

A graph of $v(\zeta)$ is given in Figure 6. Note that if $u_{B}=1$, then $\zeta_{B}=0$, and that if $u_{B}=\bar{u}(\tau)$, then the rarefaction wave disappears and the solution is given by the shock $\{\bar{u}(\tau), 0\}$. 

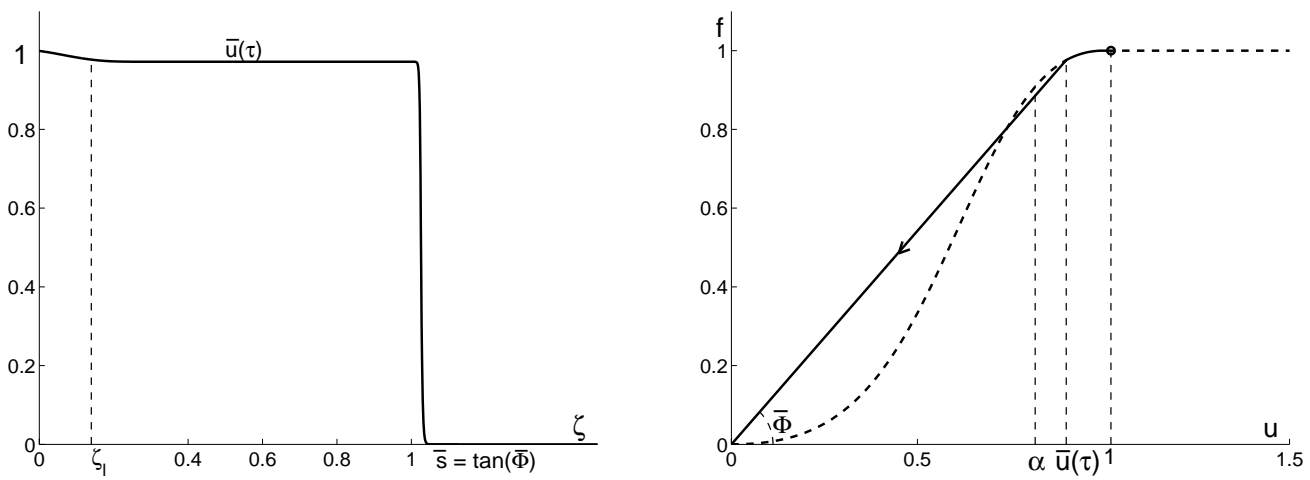

Figure 6: Case I: Solution graph (left) and flux function (right), with transitions from $u_{B}=1$ to $\bar{u}(\tau)$ and from $\bar{u}(\tau)$ to 0

Case II: $\left(u_{B}, \tau\right) \in \mathcal{B}$

It follows from Theorem 1.2 that there are no traveling wave solutions with $u_{\ell}=u_{B}$ and $u_{r}=0$, so that the shock $\left\{u_{B}, 0\right\}$ is now not admissible. However, in Theorem 1.3 we have shown that there does exist a traveling wave solution, and hence an admissible shock, with $u_{\ell}=u_{B}$ and $u_{r}=\bar{u}(\tau)$, and speed $s=s\left(u_{B}, \bar{u}(\tau)\right)$. This shock is then followed by a shock from $u=\bar{u}(\tau)$ down to $u=0$, which is admissible because by Theorem 1.1, there does exist a traveling wave solution which connects $\bar{u}$ and $u=0$ with speed $\bar{s}>s\left(u_{B}, \bar{u}(\tau)\right)$. An example of this type of solution is shown in Figure 7. The undershoot in the solution graph is due to oscillations which are also present in the travelling waves.
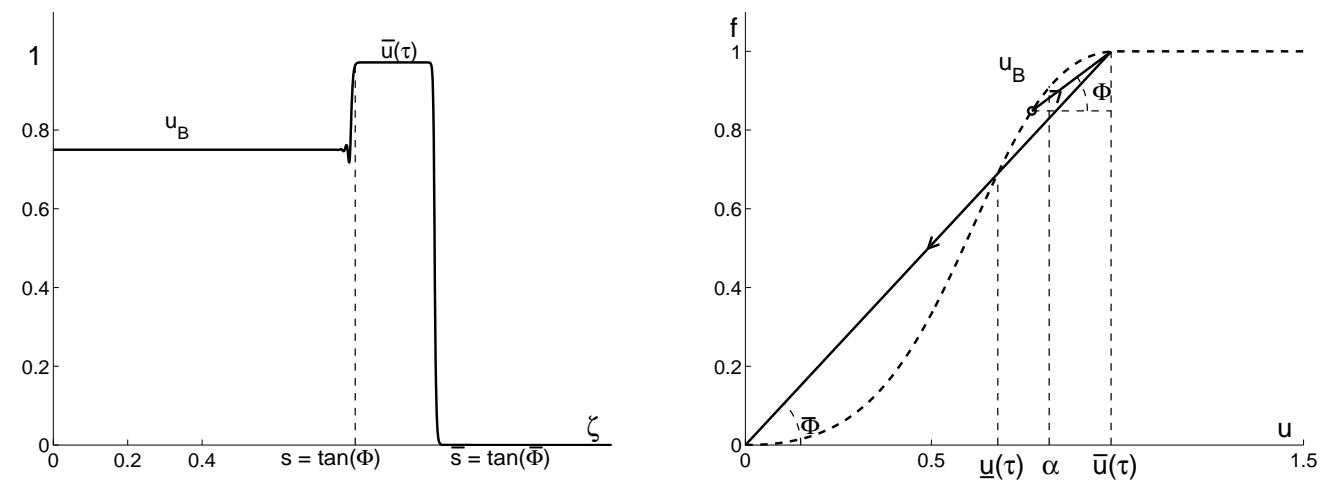

Figure 7: Case II: Solution graph (left) and flux function (right), with transitions from $u_{B}=0.75$ to $\bar{u}(\tau)$ and from $\bar{u}(\tau)$ to 0

Remark 2.1 It is readily seen that

$$
s\left(u_{B}, \bar{u}(\tau)\right) \nearrow \bar{s} \quad \text { as } \quad u_{B} \searrow \underline{u},
$$


while the plateau level $\bar{u}$ remains the same. Thus, in this limit, the plateau

$$
\left\{\left(u, \frac{x}{t}\right): u=\bar{u}(\tau), s\left(u_{B}, \bar{u}(\tau)\right)<\frac{x}{t}<\bar{s}\right\}
$$

becomes thinner and thinner and eventually disappears when $u_{B}=\underline{u}$.

Remark 2.2 If $u_{B}=1$ and $\bar{u}(\tau)>1$, then the first shock degenerates in the sense that

$$
s\left(u_{B}, \bar{u}(\tau)\right)=0 \quad \text { and } \quad u(x, t)=\bar{u}(\tau) \text { for all } \quad 0<\frac{x}{t}<\bar{s} .
$$

Case III: $\left(u_{B}, \tau\right) \in \mathcal{C}$

We have seen in Theorem 1.2 that in this case there exists a traveling wave solution with $u_{\ell}=u_{B}$ and $u_{r}=0$. It may exhibit oscillatory behaviour near $u=u_{\ell}$, and it leads to the classical entropy shock solution $\left\{u_{B}, 0\right\}$. An example of such a solution is shown in Figure 8. Note the overshoot in the solution graph, reflecting oscillations also present in the travelling waves.
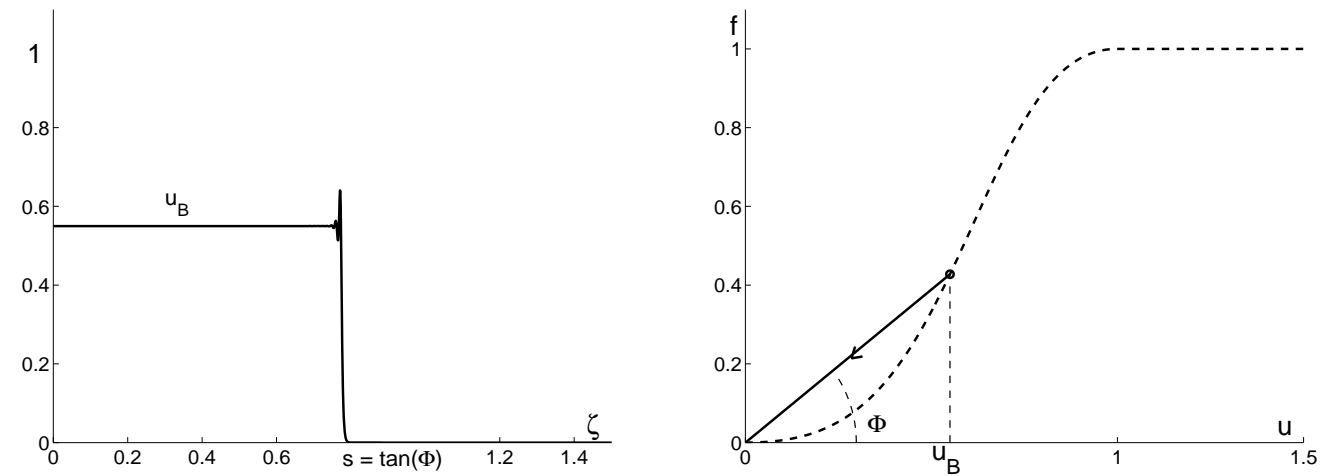

Figure 8: Case III: Solution graph (left) and flux function (right), with transition from $u_{B}=0.55$ to 0

\section{Numerical experiments for large times}

In this section we report on numerical experiments carried out for the initial-value problem for equation (1.17) in the domain $S=\mathbf{R} \times \mathbf{R}^{+}$:

$$
\begin{cases}\frac{\partial u}{\partial t}+\frac{\partial f(u)}{\partial x}=\varepsilon \frac{\partial^{2} u}{\partial x^{2}}+\varepsilon^{2} \tau \frac{\partial^{3} u}{\partial x^{2} \partial t} & \text { in } \quad S \\ u(x, 0)=u_{B} \tilde{H}(-x) & \text { for } \quad x \in \mathbf{R}\end{cases}
$$

Here $\tilde{H}(x)$ is a smooth monotone approximation of the Heaviside function $H$. Following [9], starting initially with $u_{B} H(-x)$ would cause a jump discontinuity in $u$ at $x=0$, which persists for all $t>0$. This would require an appropriate numerical approach to 
ensure the continuity in flux and pressure. By the above choice we avoid this unnecessary complication.

For solving (3.1) numerically we use the forward Euler scheme. The terms involving $\partial_{x x}$ are discretized by finite differences. To deal with the first order term we apply a minmod slope limiter method that is based on first order upwinding and Richtmyer's scheme. Even though the scheme is explicit, we have to solve a linear system for each time step. This is due to the time derivative in the last term in (3.1a). Details can be found in [7] (see also [6], Chapter 3), where alternative schemes are discussed.

Important parameters in this problem are $M, \varepsilon, \tau>0$, and $u_{B} \in(0,1]$. The scaling

$$
x \rightarrow \frac{x}{\varepsilon}, \quad t \rightarrow \frac{t}{\varepsilon}
$$

removes the parameter $\varepsilon$ from the equation (3.1a). Therefore, we fix $\varepsilon=1$ and we show how for different values of $\tau$ and $u_{B}$ the solution $u(x, t)$ of Problem (3.1) converges as $t \rightarrow \infty$ to qualitatively different final profiles. Throughout we take $M=2$. Computationally we found that (see also Figures 3 and 5)

$$
\tau_{*}=0.61 \ldots
$$

In the figures below we show graphs of solutions at various times $t$, appropriately scaled in space. Specifically, we show graphs of the function

$$
w(\zeta, t)=u(x, t) \quad \text { where } \quad \zeta=\frac{x}{t},
$$

so that a front with speed $s$ will be located at $\zeta=s$.

We begin with a simulation when $\left(u_{B}, \tau\right)=(1,0.2) \in \mathcal{A}_{1}$. In Figure 9 we show the resulting solution $w(\zeta, t)$ at time $t=1000$. It is evident that $w$ converges to the entropy solution constructed in Section 2 for $\left(u_{B}, \tau\right) \in \mathcal{A}_{1}$.

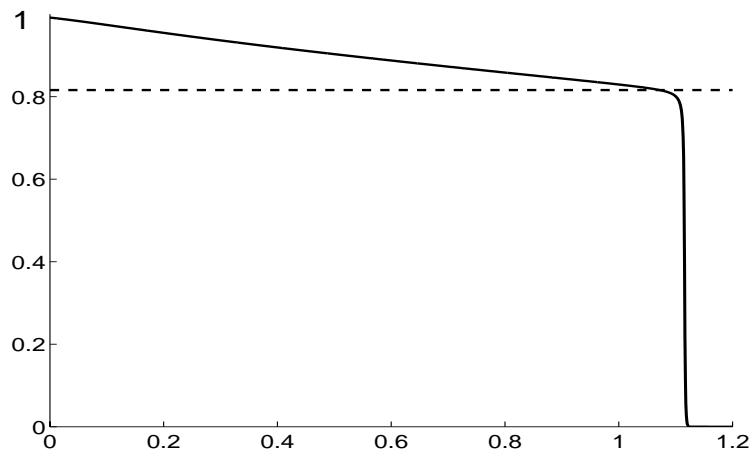

Figure 9: Graph of $w(\zeta, t)$ at $t=1000$ when $\left(u_{B}, \tau\right)=(1,0.2) \in \mathcal{A}_{1}$. In this case $\bar{u}(\tau)=0.816=\alpha$ and $s=1.11$

In the next simulation we raise $\tau$ to a value above $\tau_{*}: \tau=5$. In the first of these experiments, in which we keep $u_{B}=1$, we see that for large time the graph consists of 


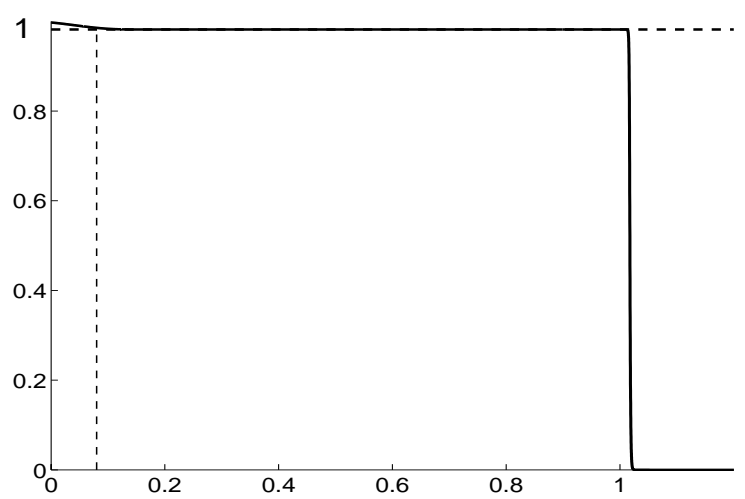

(a) $\left(u_{B}, \tau\right)=(1,5) \in \mathcal{A}_{2}$

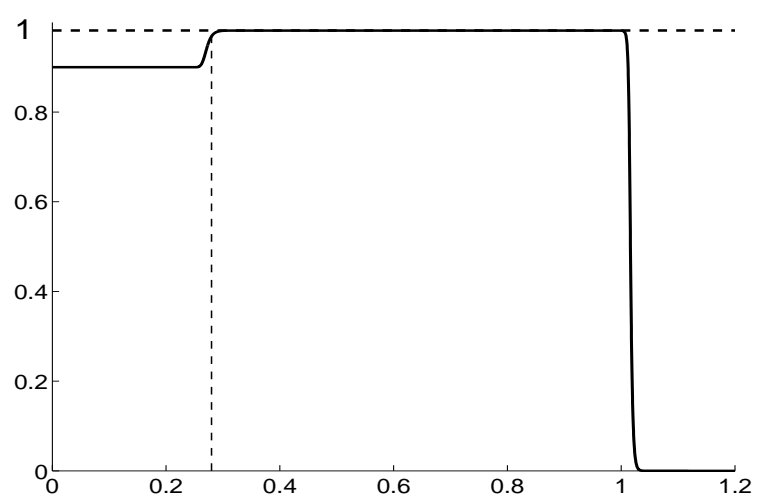

(b) $\left(u_{B}, \tau\right)=(0.9,5) \in \mathcal{B}$

Figure 10: Graphs of $w(\zeta, t)$ at $t=1000$ when $\left(u_{B}, \tau\right)=(1,5) \in \mathcal{A}_{2}$ (left) and $\left(u_{B}, \tau\right)=$ $(0.9,5) \in \mathcal{B}$ (right). Here $\bar{u}(\tau) \approx 0.98$ and $s=1.02$, while $\zeta_{\ell}=0.08$ (left) and $s_{B}=0.28$ (right)

three pieces: one in which $w$ gradually decreases from $w=u_{B}=1$ to the "plateau" value $w=\bar{u}$, one in which $w$ is constant and equal to $\bar{u}$, and one in which it drops down to $u=0$, see Figure 10a. It is clear from the graph that $\bar{u}>\alpha$. The plateau value $\bar{u} \approx 0.98$ computed here is in good agreement with the one obtained numerically when determining the graphs of $\bar{u}$ and $\underline{u}$ - see also Figures 3 and 5 .

In the next experiment we keep $\tau=5$, but we set $u_{B}=0.9$. We are then in the region $\mathcal{B}$. For large times the solution $w(\zeta, t)$ develops two shocks, one where it jumps up from $u_{B}$ to the plateau at $\bar{u}$, and one where it jumps down from $\bar{u}$ to $w=0$, see Figure 10(b).

In the next three experiments we decrease the value of $u_{B}$ to values around the value $\underline{u} \approx 0.68$. The results are shown in Figure 11, where we have zoomed into the front. We
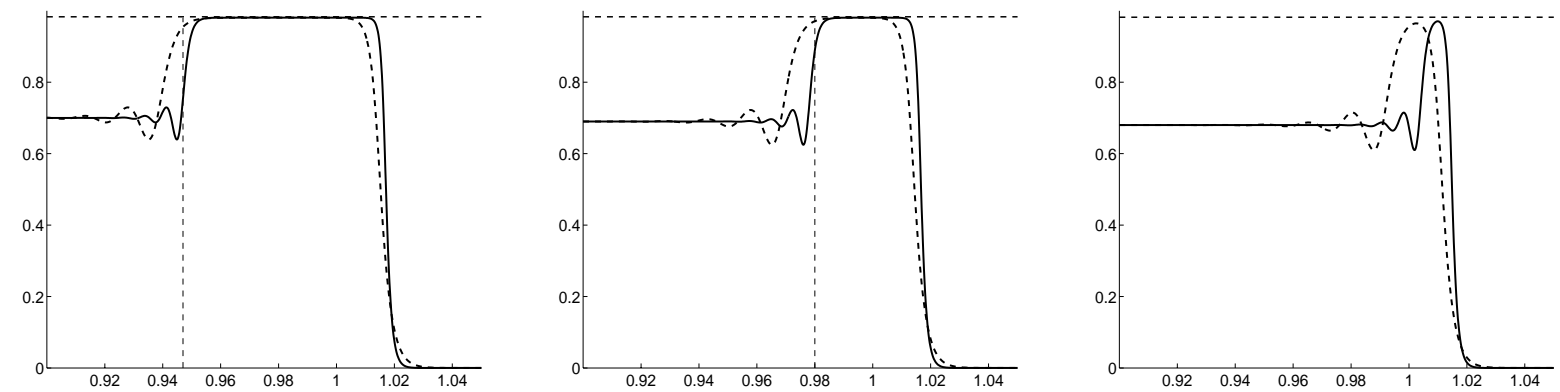

Figure 11: Graphs of $w(\zeta, t)$ with $\tau=5$ at $t=1000$ (dashed) and $t=2000$ (solid); zoomed view: $0.9 \leq \zeta \leq 1.05$. Here $\underline{u}(\tau) \approx 0.68$, and $u_{B}$ approaches $\underline{u}(\tau)$ from above through 0.70 (left), 0.69 (middle), and 0.68 (right). Then $s_{B}$ increases from 0.95 (left) to 0.98 (middle) up to 1.02 (right). The other values are $\bar{u}(\tau) \approx 0.98$ and $s=1.02$

see that, as $u_{B}$ decreases and approaches the boundary between the regions $\mathcal{B}$ and $\mathcal{C}_{2}$ in 
Figure 5 , the part of the graph where $w \approx u_{B}$ grows at the expense of the part where $w \approx \bar{u}$.

Finally, in Figure 12 we show the graph of $w(\zeta, t)$ when $\tau=5$ and $u_{B}$ is further reduced to 0.55 , so that we are now in $\mathcal{C}_{2}$. We find that the solution no longer jumps up to a higher

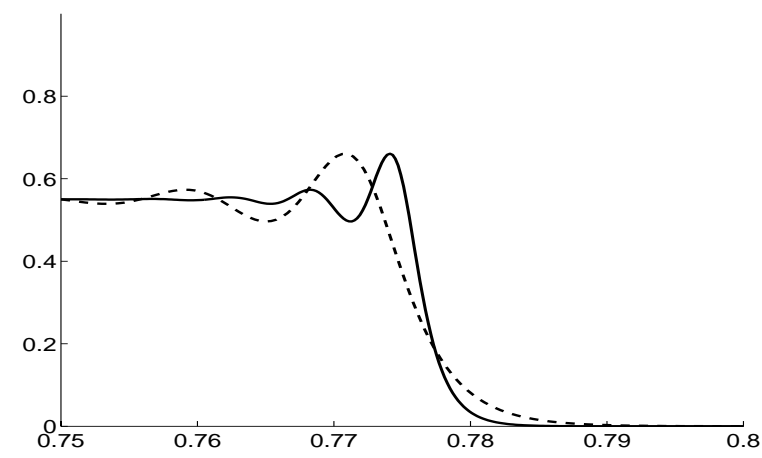

Figure 12: Graphs of $w(\zeta, t)$ at $t=1000$ (dashed) and $t=2000$ (solid) when $\left(u_{B}, \tau\right)=$ $(0.55,5) \in \mathcal{C}_{2} ;$ zoomed view: $0.75 \leq \zeta \leq 0.8$. Then $s=0.777$

plateau, but instead jumps right down after a small oscillation.

Note that the oscillations in Figures 11 and 12 contract around the shock as time progresses. This is due to the scaling, since we have plotted $w(\zeta, t)$ versus $\zeta=x / t$ for different values of time $t$.

We conclude from these simulations that the entropy solutions constructed in Section 2 emerge as limiting solutions of the Cauchy Problem (3.1). This suggests that these entropy solutions enjoy certain stability properties. It would be interesting to see whether these same entropy solutions would emerge when the initial value were chosen differently. We leave this question to a future study.

\section{$4 \quad$ Proof of Theorem 1.1}

In Theorem 1.1 we consider travelling wave solutions $u(\eta)$ of equation (1.17) in which the limiting conditions have been chosen so that $u(-\infty)=u_{\ell} \geq \alpha$ and $u(\infty)=u_{r}=0$. Putting $u_{r}=0$ in (1.18) we find that they are solutions of the problem

$$
\left(\mathrm{TW}_{0}\right)\left\{\begin{array}{c}
s \tau u^{\prime \prime}-u^{\prime}-s u+f(u)=0 \quad \text { for } \quad-\infty<\eta<\infty, \\
u(-\infty)=u_{\ell}, \quad u(+\infty)=0,
\end{array}\right.
$$

in which the speed $s$ is a-priori determined by $u_{\ell}$ through

$$
s=s\left(u_{\ell}\right) \stackrel{\text { def }}{=} \frac{f\left(u_{\ell}\right)}{u_{\ell}} .
$$

The proof proceeds in a series of steps: 
Step 1: We choose $u_{\ell} \in(\alpha, \beta)$ and prove that there exists a unique $\tau>0$ for which Problem $\left(\mathrm{TW}_{0}\right)$ has a solution, which is also unique. This defines a function $\tau=\tau\left(u_{\ell}\right)$ on $(\alpha, \beta)$. We then show that $\tau\left(u_{\ell}\right)$ is increasing, continuous and that

$$
\tau(u) \rightarrow \infty \quad \text { if } \quad u \rightarrow \beta .
$$

Finally, we write

$$
\tau_{*} \stackrel{\text { def }}{=} \lim _{u \rightarrow \alpha^{+}} \tau(u)
$$

Step 2: We show that for any $\tau \in\left(0, \tau_{*}\right]$, Problem $\left(\mathrm{TW}_{0}\right)$ has a solution with $u_{\ell}=\alpha$.

The proof is concluded by defining the function $\bar{u}_{\ell}(\tau)$ on $\left(\tau_{*}, \infty\right)$ as the inverse of the function $\tau\left(u_{\ell}\right)$ on the interval $(\alpha, \beta)$. The resulting function $\bar{u}(\tau)$, defined by (1.17) on $\mathbf{R}^{+}$, then has all the properties required in Theorem 1.1.

\subsection{The function $\tau(u)$}

As a first result we prove that $\tau(u)$ is well defined on the interval $(\alpha, \beta)$.

Lemma 4.1 For each $u_{\ell} \in(\alpha, \beta)$ there exists a unique value of $\tau$ such that there exists a solution of Problem $\left(T W_{0}\right)$. This solution is unique and decreasing.

Proof. It is convenient to write equation (4.1a) in a more conventional form, and introduce the variables

$$
\xi=-\eta / \sqrt{s \tau} \quad \text { and } \quad \bar{u}(\xi)=u(\eta)
$$

In terms of these variables, Problem $\left(\mathrm{TW}_{0}\right)$ becomes

$$
\left\{\begin{array}{c}
u^{\prime \prime}+c u^{\prime}-g(u)=0 \quad \text { in } \quad-\infty<\xi<\infty, \\
u(-\infty)=0, \quad u(+\infty)=u_{\ell},
\end{array}\right.
$$

where

$$
c=\frac{1}{\sqrt{s \tau}} \quad \text { and } \quad g(u)=s u-f(u),
$$

and the overbars have been omitted. Graphs of $g(u)$ for $M=2$ and different values of $s$ are shown in Figure 13.

We study Problem (4.3) in the phase plane and write equation (4.3a) as the first order system

$$
\mathcal{P}(c, s)\left\{\begin{array}{l}
u^{\prime}=v, \\
v^{\prime}=-c v+g(u) .
\end{array}\right.
$$

For $u_{\ell} \in(\alpha, \beta)$ the function $g(u)$ has three distinct zeros, which we denote by $u_{i}, i=0,1$ and 2 , where

$$
u_{0}=0 \quad \text { and } \quad \alpha<u_{1}<u_{2}=u_{\ell} .
$$

Plainly the points $(u, v)=\left(u_{i}, 0\right), i=0,1,2$, are the equilibrium points of $(4.5)$ with associated eigenvalues

$$
\lambda_{ \pm}=-\frac{c}{2} \pm \frac{1}{2} \sqrt{c^{2}+4 g^{\prime}\left(u_{i}\right)} .
$$



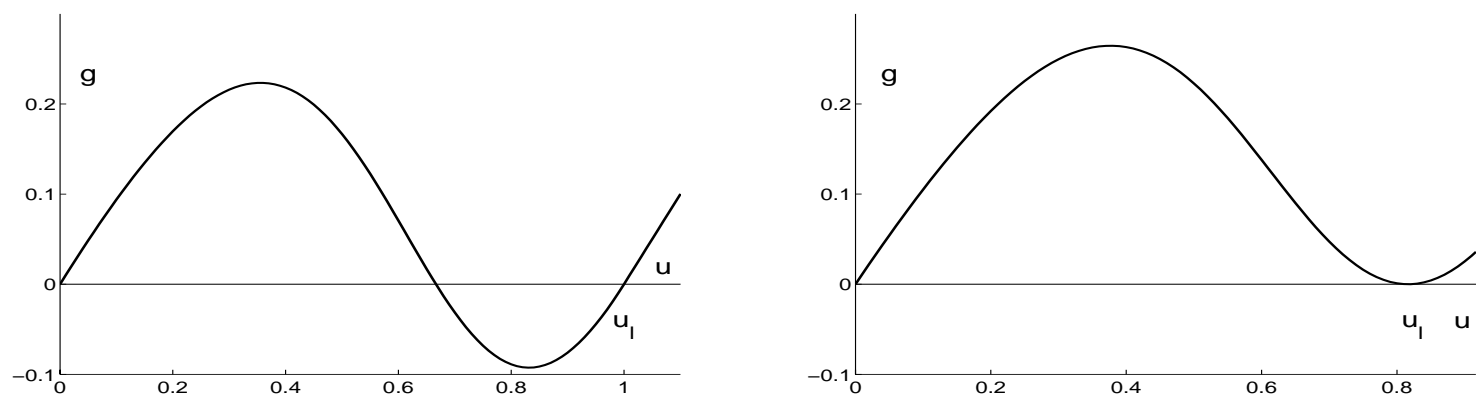

Figure 13: The function $g(u)$ for $M=2$, and $s=0.95$ (left) and $s=s(\alpha)=1.113$ (right)

Since

$$
g^{\prime}\left(u_{0}\right)>0, \quad g^{\prime}\left(u_{1}\right)<0 \quad \text { and } \quad g^{\prime}\left(u_{2}\right)>0,
$$

the outer points, $\left(u_{0}, 0\right)$ and $\left(u_{2}, 0\right)$, are saddles and $\left(u_{1}, 0\right)$ is a stable node.

Since we are interested in a travelling wave with $u(-\infty)=0$ and $u(+\infty)=u_{\ell}$, we need to investigate orbits which connect the points $(0,0)$ and $\left(u_{\ell}, 0\right)$. The existence of a unique wave speed $c$ for which there exists such a solution of the system $\mathcal{P}(c, s)$, which is unique and decreasing, has been established in [16], see also [13]. This allows us to define the function $c=c\left(u_{\ell}\right)$ for $\alpha<u_{\ell}<\beta$.

The sign of $c\left(u_{\ell}\right)$ can be determined by multiplying equation (4.3a) by $u^{\prime}$ and integrating it over $\mathbf{R}$. This yields

$$
c \int_{\mathbf{R}}\left\{u^{\prime}(\xi)\right\}^{2} d \xi=\int_{\mathbf{R}} g(u(\xi)) u^{\prime}(\xi) d \xi=\int_{0}^{u_{\ell}} g(t) d t \stackrel{\text { def }}{=} G\left(u_{\ell}\right) .
$$

Since $u_{\ell}<\beta$, it follows that $G\left(u_{\ell}\right)>0$, so that $c\left(u_{\ell}\right)>0$.

Finally, by (4.2) and (4.4), we find that $\tau$ is uniquely determined by $u_{\ell}$ through the relation

$$
\tau\left(u_{\ell}\right)=\frac{1}{s\left(u_{\ell}\right) c^{2}\left(u_{\ell}\right)} .
$$

This completes the proof of Lemma 4.1.

Lemma 4.1 allows us to define a function $\tau(u)$ on $(\alpha, \beta)$ such that if $u_{\ell} \in(\alpha, \beta)$ then Problem $\left(\mathrm{TW}_{0}\right)$ has a unique solution $u(\eta)$ if and only if $\tau=\tau\left(u_{\ell}\right)$. In the next lemma we show that the function $\tau(u)$ is strictly increasing on $(\alpha, \beta)$.

Lemma 4.2 Let $u_{\ell, i}=\gamma_{i}$ for $i=1,2$, where $\gamma_{1} \in(\alpha, \beta)$, and let $\tau\left(\gamma_{i}\right)=\tau_{i}$. Then

$$
\gamma_{1}<\gamma_{2} \quad \Longrightarrow \quad \tau_{1}<\tau_{2} \text {. }
$$

Proof. For $i=1,2$ we write

$$
s_{i}=\frac{f\left(\gamma_{i}\right)}{\gamma_{i}} \quad \text { and } \quad g_{i}(u)=s_{i} u-f(u) .
$$


Since

$$
\frac{d}{d u}\left(\frac{f(u)}{u}\right)=\frac{1}{u}\left(f^{\prime}(u)-\frac{f(u)}{u}\right)<0 \quad \text { for } \quad \alpha \leq u<\beta,
$$

it follows that

$$
\gamma_{1}<\gamma_{2} \quad \Longrightarrow \quad s_{1}>s_{2} \text { and } g_{1}(u)>g_{2}(u) \text { for } u>0 \text {. }
$$

To prove Lemma 4.2 we return to the formulation used in the proof of Lemma 4.1. Traveling waves correspond to heteroclinic orbits in the $(u, v)$-plane. Those associated with $\gamma_{1}$ and $\gamma_{2}$ we denote by $\Gamma_{1}$ and $\Gamma_{2}$. They connect the origin to respectively $\left(\gamma_{1}, 0\right)$ and $\left(\gamma_{2}, 0\right)$.

We shall show that

$$
\gamma_{1}<\gamma_{2} \quad \Longrightarrow \quad c_{1}=c\left(\gamma_{1}\right)>c\left(\gamma_{2}\right)=c_{2} .
$$

We can then conclude from (4.4) that

$$
\tau_{2} s_{2}>\tau_{1} s_{1} \quad \Longrightarrow \quad \tau_{2}>\frac{s_{1}}{s_{2}} \tau_{1}>\tau_{1}
$$

as asserted.

Thus, suppose to the contrary, that $c_{1} \leq c_{2}$. We claim that this implies that near the origin the orbit $\Gamma_{1}$ lies below $\Gamma_{2}$. Orbits of the system $\mathcal{P}(c, s)$ leave the origin along the unstable manifold under the angle $\theta$ given by

$$
\theta=\theta(c, s) \stackrel{\text { def }}{=} \frac{1}{2}\left\{\sqrt{c^{2}+4 s}-c\right\} .
$$

An elementary computation shows that

$$
\frac{\partial \theta}{\partial c}<0 \quad \text { and } \quad \frac{\partial \theta}{\partial s}>0
$$

Hence, since $s_{1}>s_{2}$ and we assume that $c_{1} \leq c_{2}$, it follows that

$$
\theta_{1}=\theta\left(c_{1}, s_{1}\right)>\theta\left(c_{2}, s_{2}\right)=\theta_{2},
$$

and hence that the orbit $\Gamma_{1}$ starts out above $\Gamma_{2}$.

Since $\left(\gamma_{2}, 0\right)$ lies to the right of the point $\left(\gamma_{1}, 0\right)$ we conclude that $\Gamma_{1}$ and $\Gamma_{2}$ must intersect. Let us denote the first point of intersection by $P=\left(u_{0}, v_{0}\right)$. Then at $P$ the slope of $\Gamma_{1}$ cannot exceed the slope of $\Gamma_{2}$. The slopes at $P$ are given by

$$
\left.\frac{d v}{d u}\right|_{\Gamma_{i}}=-c_{i}+\frac{g_{i}\left(u_{0}\right)}{v_{0}}, \quad i=1,2 .
$$

Because $g_{1}(u)>g_{2}(u)$ for $u>0$ by (4.9), it follows that

$$
\left.\frac{d v}{d u}\right|_{\Gamma_{1}}>\left.\frac{d v}{d u}\right|_{\Gamma_{2}} \quad \text { at } P,
$$

so that, at $P$, the slope of $\Gamma_{1}$ exceeds the slope of $\Gamma_{2}$, a contradiction. Therefore we find that $c_{1}>c_{2}$, as asserted.

In the next lemma we show that the function $\tau(u)$ is continuous. 
Lemma 4.3 The function $\tau:(\alpha, \beta) \rightarrow \mathbf{R}^{+}$is continuous.

Proof. Because the function $s(\gamma)=\gamma^{-1} f(\gamma)$ is continuous, it suffices to show that the function $c(\gamma)$ is continuous. Since we have shown in the proof of Lemma 4.2 that $c(\gamma)$ is decreasing (cf. (4.10)), we only need to show that it cannot have any jumps.

Suppose to the contrary that it has a jump at $\gamma_{0}$, and let us write

$$
\liminf _{\gamma \searrow \gamma_{0}^{+}} c(\gamma)=c^{+} \quad \text { and } \quad \limsup _{\gamma / \gamma_{0}^{-}} c(\gamma)=c^{-} .
$$

Then, since $c(\gamma)$ is decreasing, we may assume that $c^{-}>c^{+}$.

Thus, there exist sequences $\left\{\gamma_{n}^{-}\right\}$and $\left\{\gamma_{n}^{+}\right\}$with corresponding heteroclinic orbits $\left(u_{n}^{ \pm}, v_{n}^{ \pm}\right)$and wave speeds $c_{n}^{ \pm}$, such that

$$
c_{n}^{+} \searrow c^{+} \quad \text { and } \quad c_{n}^{-} \nearrow c^{-} \quad \text { as } n \rightarrow \infty \text {. }
$$

Since the unstable manifold at $(0,0)$ and the stable manifold at $(\gamma, 0)$ depend continuously on $c$, it follows that the corresponding orbits also converge, i.e. that there exist orbits $\left(u^{+}, v^{+}\right)$and $\left(u^{-}, v^{-}\right)$such that

$$
\left(u_{n}^{ \pm}, v_{n}^{ \pm}\right)(\xi) \rightarrow\left(u^{ \pm}, v^{ \pm}\right)(\xi) \quad \text { as } \quad n \rightarrow \infty
$$

uniformly on $\mathbf{R}$. This argument yields two heteroclinic orbits, one with speed $c^{+}$and one with speed $c^{-}$, which both connect the origin to the point $\left(\gamma_{0}, 0\right)$. Since by Lemma 4.1 there exists only one such orbit, we have a contradiction.

It follows that $c^{-}=c^{+}$, and continuity of the function $c(\gamma)$, and hence of $\tau(\gamma)$, has been established.

In the following lemma we prove the final assertion made in Step 1, which involves the behaviour of $\tau(u)$ as $u \rightarrow \beta$.

Lemma 4.4 We have

$$
\tau(\gamma) \rightarrow \infty \quad \text { as } \quad \gamma \rightarrow \beta^{-} .
$$

Proof. In view of the definition (4.7) of $\tau$, it suffices to show that $c(\gamma) \rightarrow 0$ as $\gamma \rightarrow \beta$. Proceeding as in the proof of Lemma 4.3 we find that $c(\gamma)$ and the orbit $\Gamma(c(\gamma))$ converge to, say $c_{0}$ and $\Gamma\left(c_{0}\right)=\left\{\left(u_{0}, v_{0}\right)(t): t \in \mathbf{R}\right\}$, as $\gamma \rightarrow \beta$. Note that

$$
c(\gamma) \int_{\mathbf{R}} v^{2}(\xi ; \gamma) d \xi=\int_{0}^{\gamma} g(t ; \gamma) d t
$$

where $g(t ; \gamma)=s(\gamma) t-f(t)$. If we let $\gamma \rightarrow \beta$ in this identity we obtain

$$
c_{0} \int_{\mathbf{R}} v_{0}^{2}(\xi) d \xi=\int_{0}^{\beta} g(t ; \beta) d t=0 .
$$

Because at the origin the unstable manifold points into the first quadrant when $\gamma=\beta$ (cf. (4.11)), it follows that $v_{0}>0$ on $\mathbf{R}$. Therefore, (4.13) implies that $c_{0}=0$, as asserted. 


\subsection{Traveling waves with $u_{\ell}=\alpha$}

In Lemmas 4.1 and 4.2 we have shown that $\tau(u)$ is an increasing function on $(\alpha, \beta)$. Since $\tau(u)>0$ for all $u \in(\alpha, \beta)$, the limit

$$
\tau_{*} \stackrel{\text { def }}{=} \lim _{u \rightarrow \alpha^{+}} \tau(u)
$$

exists. In the following lemmas we show that $\tau_{*}>0$ and that for all $\tau \in\left(0, \tau_{*}\right]$, Problem $\left(\mathrm{TW}_{0}\right)$ has a unique solution with $u_{\ell}=\alpha$.

Let $\mathcal{S} \in \mathbf{R}^{+}$denote the set of values of $\tau$ for which Problem $\left(\mathrm{TW}_{0}\right)$ has a unique solution with $u_{\ell}=\alpha$.

Lemma 4.5 There exists a constant $\tau_{0}>0$ such that $\left(0, \tau_{0}\right) \subset \mathcal{S}$.

Proof. We shall show that there exists a wave speed $c_{0}>0$ such that if $c>c_{0}$, then Problem (4.5) has a heteroclinic orbit connecting the origin to the point $(\alpha, 0)$. This then yields Lemma 4.5 when we put

$$
\tau_{0}=\frac{1}{c_{0}^{2} s(\alpha)} .
$$

In (4.6) we saw that the origin is a saddle and that the slope of the unstable manifold is given by

$$
\theta(c)=\frac{1}{2}\left\{\sqrt{c^{2}+4 s}-c\right\} .
$$

Note that

$$
\theta(c)<\frac{1}{c} g^{\prime}(0)=\frac{s}{c}
$$

Hence, near the origin the orbit lies below the isocline $\mathcal{I}_{v}=\left\{(u, v): v=c^{-1} g(u), u \in \mathbf{R}\right\}$.

Since $u^{\prime}>0$ and $v^{\prime}>0$ in the lens shaped region

$$
\mathcal{L}=\left\{(u, v): 0<u<\alpha, 0<v<c^{-1} g(u), u \in \mathbf{R}\right\},
$$

the orbit will leave $\mathcal{L}$ again. To see what happens next, we consider the triangular region $\Omega_{m}$ bounded by the positive $u$ - and $v$-axis, and the line

$$
\ell_{m} \stackrel{\text { def }}{=}\{(u, v): v=m(\alpha-u)\}, \quad m>0 .
$$

On the axes the vector field points into $\Omega_{m}$, and on the line $\ell_{m}$ it points inwards if

$$
\left.\frac{d v}{d u}\right|_{\ell_{m}}=-c+\frac{g(u)}{m(\alpha-u)}<-m .
$$

Let

$$
m_{0}=\inf \{m>0: g(u)<m(\alpha-u) \text { on }(0, \alpha)\} \text {. }
$$

Then

$$
-c+\frac{g(u)}{m(\alpha-u)} \leq-c+\frac{m_{0}}{m},
$$


and (4.14) will hold for values of $c$ and $m$ which satisfy the inequality

$$
-c+\frac{m_{0}}{m}<-m
$$

or

$$
c>m+\frac{m_{0}}{m} .
$$

To obtain the largest range of values of $c$ for which the vector field points into $\Omega_{m}$ we choose $m$ so that the right hand side of this inequality becomes smallest, i.e. we put $m=\sqrt{m_{0}}$. We thus find that for

$$
c>c_{0} \stackrel{\text { def }}{=} 2 \sqrt{m_{0}}
$$

the region $\Omega_{\sqrt{m_{0}}}$ is invariant, and hence, that the orbit must tend to the point $(\alpha, 0)$. This completes the proof of Lemma 4.5

The next lemma gives the structure of the set $\mathcal{S}$.

Lemma 4.6 If $\tau_{0} \in \mathcal{S}$, then $\left(0, \tau_{0}\right] \subset \mathcal{S}$.

Proof. As in earlier lemmas we prove a related result for Problem (4.5). Let $\mathcal{S}^{*}$ be the set of values of $c$ for which there exists a heteroclinic orbit of Problem $(4.5)$ from $(0,0)$ to $(\alpha, 0)$. We show that if $c_{0} \in \mathcal{S}^{*}$, then $\left[c_{0}, \infty\right) \subset \mathcal{S}^{*}$. Plainly this implies Lemma 4.6 with $\tau_{0}=1 /\left(c_{0} s^{2}\right)$.

As before, we denote the orbit emanating from the origin by $\Gamma(c)$. Suppose that $c>c_{0}$. Then, since $\theta^{\prime}(c)<0$ it follows that $\theta\left(c_{0}\right)>\theta(c)$, so that near the origin $\Gamma\left(c_{0}\right)$ lies above $\Gamma(c)$. We claim that $\Gamma\left(c_{0}\right)$ and $\Gamma(c)$ will not intersect for $u \in(0, \alpha)$. Accepting this claim for the moment, we conclude that since $\Gamma\left(c_{0}\right)$ tends to $(\alpha, 0)$, the orbit $\Gamma(c)$ must converge to $(\alpha, 0)$ as well.

It remains to prove the claim. Suppose that $\Gamma\left(c_{0}\right)$ and $\Gamma(c)$ do intersect at some $u \in(0, \alpha)$, and let $\left(u_{0}, v_{0}\right)$ be the first point of intersection. Then

$$
\left.\frac{d v}{d u}\right|_{\Gamma(c)} \geq\left.\frac{d v}{d u}\right|_{\Gamma\left(c_{0}\right) \quad \text { at } \quad\left(u_{0}, v_{0}\right) .}
$$

But, from the differential equations we deduce that

$$
\left.\frac{d v}{d u}\right|_{\Gamma(c)}=-c+\frac{g\left(u_{0}\right)}{v_{0}}<-c_{0}+\frac{g\left(u_{0}\right)}{v_{0}}=\left.\frac{d v}{d u}\right|_{\Gamma\left(c_{0}\right)} \quad \text { at } \quad\left(u_{0}, v_{0}\right)
$$

which contradicts (4.16). This proves the claim and so completes the proof of Lemma 4.6.

We conclude this section by showing that $\tau_{*} \in \mathcal{S}$, and hence that $\mathcal{S}=\left(0, \tau_{*}\right]$.

Lemma 4.7 We have $\mathcal{S}=\left(0, \tau_{*}\right]$. 
Proof. It follows from Lemmas 4.1 and 4.2 that for every $\varepsilon \in(0, \beta-\alpha)$, there a exists a $\tau_{\varepsilon}=\tau(\alpha+\varepsilon)>0$ such that Problem $\left(\mathrm{TW}_{0}\right)$ has a unique traveling wave $u_{\varepsilon}(\eta)$ with speed $s_{\varepsilon}=s(\alpha+\varepsilon)$, such that

$$
u_{\varepsilon}(-\infty)=\alpha+\varepsilon \quad \text { and } \quad u_{\varepsilon}(\infty)=0
$$

This wave corresponds to a heteroclinic orbit $\Gamma_{\varepsilon}=\left\{\left(u_{\varepsilon}(\xi), v_{\varepsilon}(\xi)\right): \xi \in \mathbf{R}\right\}$ of the system $\mathcal{P}\left(c_{\varepsilon}, s_{\varepsilon}\right)$ where $c_{\varepsilon}=1 / \sqrt{s_{\varepsilon} \tau_{\varepsilon}}$, which connects the points $(0,0)$ and $(\alpha+\varepsilon, 0)$. It leaves the origin along the stable manifold under an angle $\theta_{\varepsilon}=\theta\left(c_{\varepsilon}, s_{\varepsilon}\right)$ and enters the point $(\alpha+\varepsilon, 0)$ along the stable manifold under the angle

$$
\psi_{\varepsilon}=\psi\left(c_{\varepsilon}, s_{\varepsilon}\right)=\frac{1}{2}\left\{-c_{\varepsilon}-\sqrt{c_{\varepsilon}^{2}+4 g^{\prime}(\alpha+\varepsilon)}\right\} \rightarrow-c_{0}=-\frac{1}{\sqrt{s(\alpha) \tau_{*}}} \quad \text { as } \quad \varepsilon \rightarrow 0 .
$$

Reversing time, i.e., replacing $\xi$ by $-\xi$, we can view $\Gamma_{\varepsilon}$ as the unique orbit emanating from the point $(\alpha+\varepsilon, 0)$ into the first quadrant, and entering the origin as $\xi \rightarrow \infty$. In the limit, as $\varepsilon \rightarrow 0$, we find that

$$
u_{\varepsilon}(\xi) \rightarrow u_{0}(\xi) \quad \text { and } \quad v_{\varepsilon}(\xi) \rightarrow v_{0}(\xi) \quad \text { as } \quad \varepsilon \rightarrow 0 \quad \text { for } \quad-\infty<\xi \leq \xi_{0},
$$

where $\xi_{0}$ is any finite number. We claim that

$$
u_{0}(\xi) \rightarrow 0 \quad \text { and } \quad v_{0}(\xi) \rightarrow 0 \quad \text { as } \quad \xi \rightarrow \infty
$$

i.e., $\Gamma_{0} \stackrel{\text { def }}{=}\left\{\left(u_{0}(\xi), v_{0}(\xi)\right): \xi \in \mathbf{R}\right\}$ is a heteroclinic orbit, which connects $(\alpha, 0)$ and the origin $(0,0)$.

Suppose to the contrary that $\Gamma_{0}$ does not enter the origin as $\xi \rightarrow \infty$, and possibly not even exist for all $\xi \in \mathbf{R}$. Then, since

$$
\frac{d v}{d u}=-c_{0}+\frac{g(u)}{v}>-c_{0} \quad \text { if } \quad 0<u<\alpha, \quad v>0
$$

$\Gamma_{0}$ must leave the first quadrant in finite time, either through the $u$-axis or through the $v$-axis. This means by continuity that for $\varepsilon$ small enough $\Gamma_{\varepsilon}$ must also leave the first quadrant in finite time. Since $\Gamma_{\varepsilon}$ is known to enter the origin for every $\varepsilon>0$, and hence never to leave the first quadrant, we have a contradiction. This proves the claim that $\Gamma_{0}$ is a heteroclinic orbit, which connects $(\alpha, 0)$ and $(0,0)$.

\section{$5 \quad$ Proof of Theorems 1.2 and 1.3}

For the proofs of Theorems 1.2 and 1.3 we turn to the system $\mathcal{P}(c, s)$ defined in Section 4. For convenience we restate it here

$$
\mathcal{P}(c, s)\left\{\begin{array}{l}
u^{\prime}=v, \\
v^{\prime}=-c v+g_{s}(u),
\end{array}\right.
$$

where

$$
c=\frac{1}{\sqrt{s \tau}} \quad \text { and } \quad g_{s}(u)=s u-f(u) .
$$

Part (a) of Theorem 1.2 is readily seen to be a consequence of the following lemma: 
Lemma 5.1 Let $\tau>\tau_{*}$ be given. Then for every $u_{\ell} \in(0, \underline{u})$, there exists a unique heteroclinic orbit of the system $\mathcal{P}(c, s)$ in which

$$
s=s_{\ell}=\frac{f\left(u_{\ell}\right)}{u_{\ell}} \quad \text { and } \quad c=c_{\ell}=\frac{1}{\sqrt{s_{\ell} \tau}}
$$

which connects $(0,0)$ and $\left(u_{\ell}, 0\right)$.

Proof. Let $\Gamma_{\ell}$ and $\bar{\Gamma}$ denote the orbits of $\mathcal{P}\left(c_{\ell}, s_{\ell}\right)$ and $\mathcal{P}(\bar{c}, \bar{s})$, which enter the first quadrant from the origin. They do this under the angles $\theta\left(c_{\ell}, s_{\ell}\right)$ and $\theta(\bar{c}, \bar{s})$, respectively. Since $c_{\ell}>\bar{c}$ and $s_{\ell}<\bar{s}$, it follows from (4.12) that

$$
\theta\left(c_{\ell}, s_{\ell}\right)<\theta(\bar{c}, \bar{s}) \text {. }
$$

Hence, near the origin, $\Gamma_{\ell}$ lies below $\bar{\Gamma}$. Thus, $\Gamma_{\ell}$ enters the region $\Omega$ enclosed between $\bar{\Gamma}$ and the $u$-axis. Since

$$
\left.\frac{d v}{d u}\right|_{\Gamma_{\ell}}=-c_{\ell}+\frac{s_{\ell} u-f(u)}{v}<-\bar{c}+\frac{\bar{s} u-f(u)}{v}=\left.\frac{d v}{d u}\right|_{\bar{\Gamma}}
$$

it follows that $\Gamma_{\ell}$ cannot leave $\Omega$ though its "top" $\bar{\Gamma}$. We define the following subsets of the bottom of $\Omega$ :

$$
\begin{aligned}
& S_{1}=\left\{(u, v): 0<u<u_{\ell}, v=0\right\}, \\
& S_{2}=\left\{(u, v): u=u_{\ell}, v=0\right\}, \\
& S_{3}=\left\{(u, v): u_{\ell}<u<\bar{u}, v=0\right\} .
\end{aligned}
$$

Inspection of the vector field show that orbits can only leave $\Omega$ through $S_{3}$. Note that the set $S_{2}$ consists of an equilibrium point.

There are two possibilities: either $\Gamma_{\ell}$ never leaves $\Omega$, or $\Gamma_{\ell}$ leaves $\Omega$, necessarily through the set $S_{3}$. In the first case $\Gamma_{\ell}$ is a heteroclinic orbit from $(0,0)$ to $\left(u_{\ell}, 0\right)$, and the proof is complete.

Thus, let us assume that $\Gamma_{\ell}$ leaves $\Omega$ at some point $(u, v)=\left(u_{0}, 0\right)$. Consider the energy function

$$
\mathcal{H}(u, v)=\frac{1}{2} v^{2}-G_{s_{\ell}}(u) .
$$

and write $H(x)=\mathcal{H}(u(x), v(x))$, when $(u(x), v(x))$ is an orbit. Then differentiation shows that

$$
H^{\prime}(x)=-c_{\ell} v^{2}(x)<0 .
$$

Since $\mathcal{H}(0,0)=0$, it follows that

$$
\mathcal{H}\left(u_{0}, 0\right)=-G_{s_{\ell}}\left(u_{0}\right)<0
$$

and that

$$
\mathcal{H}(u(x), v(x))=\frac{1}{2} v^{2}-G_{s_{\ell}}(u)<-G_{s_{\ell}}\left(u_{0}\right) \quad \text { for } \quad x>x_{0} .
$$

This means that

$$
G_{s_{\ell}}(u)>G_{s_{\ell}}\left(u_{0}\right)>0 \quad \text { for } \quad x>x_{0}
$$


Let

$$
u_{1}=\inf \left\{s \in \mathbf{R}: G_{s_{\ell}}(s)>G_{s_{\ell}}\left(u_{0}\right) \text { on }\left(s, u_{0}\right)\right\} .
$$

Since $G_{s_{\ell}}\left(u_{0}\right)>0$ it follows that $u_{1} \in\left(0, u_{\ell}\right)$. Therefore

$$
\left.\begin{array}{l}
0<u_{1}<u(x)<u_{0} \\
v^{2}(x)<2\left\{G_{s_{\ell}}\left(u_{\ell}\right)-G_{s_{\ell}}\left(u_{0}\right)\right\}
\end{array}\right\} \quad \text { for } \quad x>x_{0} .
$$

From a simple energy argument we conclude that $(u(x), v(x)) \rightarrow\left(u_{\ell}, 0\right)$ as $x \rightarrow \infty$. This completes the proof of Lemma 5.1.

Part (b) follows from the following result.

Lemma 5.2 Let $\tau>\tau_{*}$ be given. For any $u_{\ell} \in(\underline{u}(\tau), \bar{u}(\tau))$ there exists no solution of the system $\mathcal{P}\left(c_{\ell}, s_{\ell}\right)$, with

$$
s_{\ell}=\frac{f\left(u_{\ell}\right)}{u_{\ell}} \quad \text { and } \quad c_{\ell}=\frac{1}{\sqrt{s_{\ell} \tau}}
$$

which connects $(0,0)$ and $\left(u_{\ell}, 0\right)$.

Proof. Let $\bar{\Gamma}$ denote the orbit corresponding to $\bar{c}$ and $\bar{s}$, which connects $(0,0)$ and the point $(\bar{u}, 0)$, and let $\Gamma_{\ell}$ denote the orbit which corresponds to $c_{\ell}$ and $s_{\ell}$. Observe that

$$
s_{\ell}>\bar{s} \quad \text { and } \quad c_{\ell}<\bar{c}
$$

and hence

$$
\theta\left(c_{\ell}, s_{\ell}\right)>\theta(\bar{c}, \bar{s})
$$

Therefore, near the origin, $\Gamma_{\ell}$ lies above $\bar{\Gamma}$. Hence, to reach the point $\left(u_{\ell}, 0\right)$, the orbit $\bar{\Gamma}_{\ell}$ has to cross $\bar{\Gamma}$ somewhere, and at the first point of crossing we must have

$$
\left.\frac{d v}{d u}\right|_{\Gamma} \geq\left.\frac{d v}{d u}\right|_{\Gamma_{\ell}}
$$

However, by the equations, we have

$$
\left.\frac{d v}{d u}\right|_{\bar{\Gamma}}=-\bar{c}+\frac{g_{\bar{s}}(u)}{u}<-c_{\ell}+\frac{g_{s_{\ell}}(u)}{u}=\left.\frac{d v}{d u}\right|_{\Gamma_{\ell}},
$$

so that we have a contradiction.

This completes the proof of Theorem 1.2

The proof of Theorem 1.3 is entirely analogous to that of Theorem 1.2, and we omit it. 


\section{Entropy dissipation}

In this section we study the Cauchy Problem

$$
(\mathrm{CP}) \begin{cases}u_{t}+(f(u))_{x}=\mathcal{A}_{\varepsilon}(u) & \text { in } \quad S=\mathbf{R} \times \mathbf{R}^{+}, \\ u(\cdot, 0)=u_{0}(\cdot) & \text { on } \quad \mathbf{R},\end{cases}
$$

where

$$
\mathcal{A}_{\varepsilon}(u)=\varepsilon u_{x x}+\varepsilon^{2} \tau u_{x x t}, \quad(\varepsilon>0) .
$$

With this choice (6.1a) becomes the regularized Buckley-Leverett equation (1.17) for which we obtained traveling wave solutions in the previous sections. In (6.1a) and (6.2) we introduce subscripts to denote partial derivatives. Without further justification we assume that Problem (CP) has a smooth, nonnegative and bounded solution $u^{\varepsilon}$ for each $\varepsilon>0$, and that there exist a limit function $u: S \rightarrow[0, \infty)$ such that for each $(x, t) \in S$,

$$
u^{\varepsilon}(x, t) \rightarrow u(x, t) \quad \text { as } \quad \varepsilon \rightarrow 0 .
$$

In addition we assume the following structural properties:

(i) For each fixed $t>0$,

$$
\begin{aligned}
& u^{\varepsilon}(x, t) \rightarrow u_{\ell} \in \mathbf{R}^{+} \quad \text { as } \quad x \rightarrow-\infty, \\
& u^{\varepsilon}(x, t) \rightarrow u_{r} \in \mathbf{R}^{+} \quad \text { as } \quad x \rightarrow+\infty \text {. }
\end{aligned}
$$

(ii) The partial derivatives of $u^{\varepsilon}$ vanish as $|x| \rightarrow \infty$.

(iii) Let $U(s)=\frac{1}{2} s^{2}$ for $s \geq 0, U_{\ell}=U\left(u_{\ell}\right)$ and $U_{r}=U\left(u_{r}\right)$. Then there exists a smooth function $\lambda_{\varepsilon}:[0, \infty) \rightarrow \mathbf{R}$ which is uniformly bounded with respect to $\varepsilon>0$ in any bounded interval $(0, T)$, such that

$$
\int_{\mathbf{R}}\left\{U\left(u^{\varepsilon}(x, t)\right)-G_{\varepsilon}(x, t)\right\} d x=0 \quad \text { for all } \quad t>0,
$$

where $G_{\varepsilon}$ is the step function

$$
G_{\varepsilon}(x, t)=U_{\ell}+\left(U_{r}-U_{\ell}\right) H\left(x-\lambda_{\varepsilon}(t)\right), \quad(x, t) \in S
$$

in which $H$ denotes the Heaviside function.

Note that the traveling waves constructed in this paper all have these properties. The main purpose of this section is to show that $U\left(u^{\varepsilon}\right)$ is an entropy for equation (6.1a) (see also [17]).

For completeness we recall some definitions. We say that the term $\mathcal{A}_{\varepsilon}(u)$ is conservative if

$$
\lim _{\varepsilon \rightarrow 0^{+}} \int_{S} \mathcal{A}_{\varepsilon}\left(u^{\varepsilon}\right) \varphi=0 \quad \text { for all } \quad \varphi \in C_{0}^{\infty}(S)
$$

and we say that $\mathcal{A}_{\varepsilon}(u)$ is entropy dissipative (for an entropy $U$ ) if

$$
\limsup _{\varepsilon \rightarrow 0^{+}} \int_{S} \mathcal{A}_{\varepsilon}\left(u^{\varepsilon}\right) U^{\prime}\left(u^{\varepsilon}\right) \varphi \leq 0 \quad \text { for all } \quad \varphi \in C_{0}^{\infty}(S), \quad \varphi \geq 0 .
$$

We establish the following theorem: 
Theorem 6.1 Let $u^{\varepsilon}$ be the solution of Problem (CP), and let $u^{\varepsilon}$ satisfy (i), (ii) and (iii). Then, the regularization $\mathcal{A}_{\varepsilon}(u)$ defined in (6.2) has the following properties:

(a) $\mathcal{A}_{\varepsilon}(u)$ is conservative.

(b) $\mathcal{A}_{\varepsilon}(u)$ is entropy dissipative for the entropy $U(u)=\frac{1}{2} u^{2}$.

Proof. Part (a). For any $\varphi \in C_{0}^{\infty}(S)$ we obtain after partial integration with respect to $x$ and $t$,

$$
\int_{S} \mathcal{A}_{\varepsilon}\left(u^{\varepsilon}\right) \varphi=\varepsilon \int_{S} u^{\varepsilon} \varphi_{x x}-\varepsilon^{2} \tau \int_{S} u^{\varepsilon} \varphi_{x x t} \rightarrow 0 \quad \text { as } \quad \varepsilon \rightarrow 0 .
$$

Part (b). To simplify notation, we drop the superscript $\varepsilon$ from $u^{\varepsilon}$. When we multiply (6.1a) by $u$ we obtain

$$
\partial_{t} U(u)+\partial_{x} F(u)=u \mathcal{A}_{\varepsilon}(u)=\varepsilon u u_{x x}+\varepsilon^{2} \tau u u_{x x t}
$$

where

$$
F(u)=\int_{0}^{u} U^{\prime}(s) f^{\prime}(s) d s=\int_{0}^{u} s f^{\prime}(s) d s=u f(u)-\int_{0}^{u} f(s) d s .
$$

An elementary computation shows that

$$
\begin{aligned}
\varepsilon u u_{x x} & =\varepsilon U_{x x}-\varepsilon u_{x}^{2} \\
\varepsilon^{2} \tau u u_{x x t} & =\varepsilon^{2} \tau\left(U_{x x t}-\frac{1}{2}\left(u_{x}^{2}\right)_{t}-\left(u_{x} u_{t}\right)_{x}\right) .
\end{aligned}
$$

Hence

$$
\begin{aligned}
\int_{S} \mathcal{A}_{\varepsilon}(u) u \varphi= & \varepsilon \int_{S} U \varphi_{x x}-\varepsilon \int_{S} u_{x}^{2} \varphi \\
& -\varepsilon^{2} \tau \int_{S} U \varphi_{x x t}+\frac{1}{2} \varepsilon^{2} \tau \int_{S} u_{x}^{2} \varphi_{t}+\varepsilon^{2} \tau \int_{S} u_{t} u_{x} \varphi_{x}
\end{aligned}
$$

Plainly

$$
\varepsilon \int_{S} U \varphi_{x x} \rightarrow 0 \quad \text { and } \quad \varepsilon^{2} \tau \int_{S} U \varphi_{x x t} \rightarrow 0 \quad \text { as } \quad \varepsilon \rightarrow 0 .
$$

Since $\varphi \geq 0$, it remains to estimate the last two terms in the right hand side of (6.7).

For this purpose we establish the following two estimates:

Lemma 6.1 Let $T>0$, and let $S_{T}=\mathbf{R} \times(0, T]$. Then there exists a constant $C>0$ such that for all $\varepsilon>0$,

$$
\varepsilon \int_{S_{T}} u_{x}^{2} \leq C
$$

and

$$
\varepsilon \int_{S_{T}} u_{t}^{2} \leq C
$$


Proof of (6.8). We write (6.5) as

$$
\partial_{t} U(u)+\partial_{x} F(u)=\varepsilon U_{x x}-\varepsilon u_{x}^{2}+\varepsilon^{2} \tau\left\{U_{x x t}-\frac{1}{2}\left(u_{x}^{2}\right)_{t}-\left(u_{t} u_{x}\right)_{x}\right\} .
$$

Using properties $(i)-(i i i)$, and writing $F_{\ell}=F\left(u_{\ell}\right), F_{r}=F\left(u_{r}\right)$, we find that

$$
\begin{aligned}
\frac{d}{d t} \int_{\mathbf{R}}\{U(x, t) & \left.-G_{\varepsilon}(x, t)\right\} d x-\frac{d \lambda_{\varepsilon}}{d t}\left(U_{r}-U_{\ell}\right) \\
& +\left(F_{r}-F_{\ell}\right)+\varepsilon \int_{\mathbf{R}} u_{x}^{2}+\frac{1}{2} \varepsilon^{2} \tau \frac{d}{d t} \int_{\mathbf{R}} u_{x}^{2} \leq 0
\end{aligned}
$$

or, when we integrate over $(0, T)$

$$
-\left\{\lambda_{\varepsilon}(t)-\lambda_{\varepsilon}(0)\right\}\left(U_{r}-U_{\ell}\right)+\left(F_{r}-F_{\ell}\right) t+\varepsilon \int_{S_{T}} u_{x}^{2}+\frac{1}{2} \varepsilon^{2} \tau \int_{\mathbf{R}} u_{x}^{2}(t) \leq \frac{1}{2} \varepsilon^{2} \tau \int_{\mathbf{R}}\left(u_{0}^{\prime}\right)^{2},
$$

from which (6.8) immediately follows.

Proof of (6.9). We multiply (6.1) by $u_{t}$. This yields

$$
u_{t}^{2}+(f(u))_{x} u_{t}=u_{t} \mathcal{A}_{\varepsilon}(u)=\varepsilon u_{t} u_{x x}+\varepsilon^{2} \tau u_{t} u_{x x t}
$$

Using the identities

$$
u_{t} u_{x x}=\left(u_{x} u_{t}\right)_{x}-\frac{1}{2}\left(u_{x}^{2}\right)_{t} \quad \text { and } \quad u_{t} u_{x x t}=\left(u_{x t} u_{t}\right)_{x}-\left(u_{x t}\right)^{2},
$$

we find that

$$
u_{t}^{2}+\frac{\varepsilon}{2}\left(u_{x}^{2}\right)_{t} \leq-f^{\prime}(u) u_{t} u_{x}+\varepsilon\left(u_{x} u_{t}\right)_{x}+\varepsilon^{2} \tau\left(u_{x t} u_{t}\right)_{x} .
$$

When we integrate over $\mathbf{R}$ and use Schwarz's inequality and properties $(i)$ and $(i i)$, we obtain

$$
\int_{\mathbf{R}} u_{t}^{2}+\frac{\varepsilon}{2} \frac{d}{d t} \int_{\mathbf{R}} u_{x}^{2} \leq \frac{1}{2} \int_{\mathbf{R}} u_{t}^{2}+\frac{K^{2}}{2} \int_{\mathbf{R}} u_{x}^{2}
$$

where $K=\max \left\{\left|f^{\prime}(s)\right|: s>0\right\}$. Hence, when we integrate over $(0, t)$,

$$
\int_{S_{t}} u_{t}^{2} \leq \varepsilon \int_{\mathbf{R}}\left(u_{0}^{\prime}\right)^{2}+K^{2} \int_{S_{t}} u_{x}^{2}
$$

In view of the first estimate this establishes (6.9), and completes the proof of Lemma 6.1.

We now return to the proof of Part (b) of Theorem 6.1. For each $\varphi \in C_{0}^{\infty}(S)$ we choose $T>0$ so that $\operatorname{supp} \varphi \subset S_{T}$. Then (6.8) implies that

$$
\varepsilon^{2} \int_{S_{T}} u_{x}^{2} \varphi_{t} \leq \varepsilon^{2} K_{1} \int_{S_{T}} u_{x}^{2} \leq \varepsilon K_{1} C \quad \text { with } \quad K_{1}=\sup \left|\varphi_{t}\right|,
$$

and (6.8) and (6.9) together imply that

$$
\varepsilon^{2} \int_{S_{T}} u_{t} u_{x} \varphi_{x} \leq \varepsilon^{2} K_{2} \int_{S_{T}}\left|u_{t}\right|\left|u_{x}\right| \leq \varepsilon K_{2} C \quad \text { with } \quad K_{2}=\sup \left|\varphi_{x}\right| .
$$


Using (6.12) and (6.13) in (6.7) we conclude that, writing $u=u^{\varepsilon}$ again,

$$
\limsup _{\varepsilon \searrow 0} \int_{S} \mathcal{A}_{\varepsilon}\left(u^{\varepsilon}\right) u^{\varepsilon} \varphi \leq 0
$$

which is what was claimed in Theorem 6.1.

It now follows from (6.5) that in the limit as $\varepsilon \rightarrow 0$,

$$
\partial_{t} U(u)+\partial_{x} F(u) \leq 0
$$

holds in a weak or distributional sense. This shows that $(U, F)$ is an entropy pair for equation (1.1).

The inequality in (6.14) indicates entropy dissipation. Across shocks $\left\{u_{\ell}, u_{r}\right\}$ it can be computed explicitly. Let

$$
u(x, t)=\left\{\begin{array}{lll}
u_{\ell} & \text { for } & x<s t \\
u_{r} & \text { for } & x>s t
\end{array}\right.
$$

Then (6.14) implies that

$$
-s\left(U_{r}-U_{\ell}\right)+\left(F_{r}-F_{\ell}\right) \leq 0 .
$$

Hence the entropy dissipation is given by

$$
E\left(u_{\ell}, u_{r}\right) \stackrel{\text { def }}{=}-s\left(U_{r}-U_{\ell}\right)+\left(F_{r}-F_{\ell}\right) .
$$

We conclude by observing that if $u=u(\eta)$ is a travelling wave satisfying Problem (TW), then $(6.15)$ can be written as

$$
E\left(u_{\ell}, u_{r}\right)=\int_{\mathbf{R}}\left\{-s(U(u))^{\prime}+(F(u))^{\prime}\right\} d \eta .
$$

Applying (6.6) and the definition of $U$ gives

$$
E\left(u_{\ell}, u_{r}\right)=\int_{\mathbf{R}} u\left(-s+\frac{d f}{d u}\right) u^{\prime} d \eta=\int_{u_{\ell}}^{u_{r}} u\left(-s+\frac{d f}{d u}\right) d u .
$$

Rewriting further

$$
-s+\frac{d f}{d u}=\frac{d}{d u}\left(-s\left(u-u_{\ell}\right)+f(u)-f\left(u_{\ell}\right)\right),
$$

integrating (6.17) by parts, and using the Rankine-Hugoniot condition yields

$$
E\left(u_{\ell}, u_{r}\right)=\int_{u_{r}}^{u_{\ell}}\left\{f(u)-f\left(u_{\ell}\right)-s\left(u-u_{\ell}\right)\right\} d u .
$$

In the special case when $u_{r}=0$ we have $s=f\left(u_{\ell}\right) / u_{\ell}$ and thus

$$
E\left(u_{\ell}, 0\right)=\int_{0}^{u_{\ell}}\{f(u)-s u\} d u \text {. }
$$

Returning to the proof of Proposition 1.1 we observe that the integral is negative provided $u_{\ell}<\beta$. Thus this condition acts as an entropy condition in the sense that $E\left(u_{\ell}, 0\right)<0$ only if $u_{\ell}<\beta$.

Acknowledgement The authors are grateful for the support from the Centrum voor Wiskunde en Informatica in Amsterdam (CWI). 


\section{References}

[1] G.I. Barenblatt, J. Garcia-Azorero, A. De Pablo and J.L. Vazquez, Mathematical model of the non-equilibrium water-oil displacement in porous strata, Applicable Anal. 65 (1997) 1945.

[2] G.I. Barenblatt, V.M. Entov and V.M. Ryzhik, Theory of Fluid Flow Through Natural Rocks, Kluwer, Dordrecht-Boston-London (1990).

[3] J. Bear, Dynamics of fluids in porous media, American Elsevier, New York, London, Amsterdam, 1972.

[4] A.L. Bertozzi, A. Münch and M. Shearer, Undercompressive shocks in thin film flows, Physica D 134 (1999) 431-464.

[5] A.L. Bertozzi and M. Shearer, Existence of undercompressive traveling waves in thin film equations, SIAM J. Math. Anal. 32 (2000) 194-213.

[6] C. Cuesta, Pseudo-parabolic equations with driving convection term, $\mathrm{PhD}$ thesis, VU Amsterdam, 2003.

[7] C. Cuesta and I.S. Pop, Long-time behaviour of a pseudo-parabolic Burgers' type equation: numerical investigation (in preparation).

[8] C. Cuesta, C.J. van Duijn and J. Hulshof, Infiltration in porous media with dynamic capillary pressure: travelling waves, Euro. J. Appl. Math. 11 (2000) 381-397.

[9] C. Cuesta and J. Hulshof, A model problem for groundwater flow with dynamic capillary pressure: stability of travelling waves, Nonlinear Anal. 52 (2003) 1199-1218.

[10] David A. DiCarlo, Experimental measurements of saturation overshoot on infiltration, Water Resour. Res. 40 (2004), W04215, doi:10.1029/2003WR002670.

[11] A.G. Egorov, R.Z. Dautov, J.L. Nieber and A.Y. Sheshukov, Stability analysis of traveling wave solution for gravity-driven flow, CMWR2002 Conf. (Delft, The Netherlands) 1: 120-127.

[12] A.G. Egorov, R.Z. Dautov, J.L. Nieber and A.Y. Sheshukov, Stability analysis of gravity-driven infiltrating flow, Water Resour. Res., 39 (2003) 1266.

[13] P.C. Fife and J.B. McLeod, The approach of solutions of nonlinear diffusion equations to travelling front solutions, Arch. Rational Mech. Anal. 65, (1977) 335-361.

[14] S.M. Hassanizadeh and W.G. Gray, Mechanics and thermodynamics of multiphase flow in porous media including interphase boundaries, Adv. Water Resour., 13 (1990) 169-186.

[15] S.M. Hassanizadeh and W.G. Gray, Thermodynamic basis of capillary pressure in porous media, Water Resources Res. 29 (1993) 3389-3405.

[16] Ya.I. Kaniel, On the stabilisation of solutions of the Cauchy problem for equations arising in the theory of combustion, Mat Sbornik 59 (1962) 245-288. See also Dokl. Akad. Nauk. SSSR 132 (1960) 268-271 (= Soviet Math. Dokl. 1 (1960) 533-536) and Dokl. Akad. Nauk. SSSR 136 (1961) 277-280 (= Soviet Math. Dokl. 2 (1961) 48-51).

[17] P.G. LeFloch, Hyperbolic systems of conservation laws. The theory of classical and nonclassical shock waves. Lectures in Mathematics ETH Zürich. Birkhäuser, Basel, 2002. 
[18] D.E. Smiles, G. Vachaud and M. Vauclin, A test of the uniqueness of the soil moisture characteristic during transient, non-hysteretic flow of water in rigid soil, Soil Sci. Soc. Am. Proc. 35 (1971) 535-539.

[19] J. Smoller, Shock waves and reaction-diffusion equations, Springer-Verlag, 1983.

[20] F. Stauffer, Time dependence of the relations between capillary pressure, water content and conductivity during drainage of porous media, LAHR Symposium on Scale Effects in Porous Media, Thessaloniki, Greece, August 29-September 1, 1978.

[21] R.E. Wilson and P. Berg, Existence and classification of travelling wave solutions to second order highway traffic models, in: Traffic and Granular Flow '01 Editors: Fukui M, Sugiyama Y, Schreckenberg M, Wolf DE pages 85-90, Springer-Verlag 2003 